\title{
A Categorification of the Ribbon Element in Quantum sl(2)
}

\author{
Anna Beliakova ${ }^{1} \cdot$ Kazuo Habiro ${ }^{2}$
}

Received: 7 July 2020 / Accepted: 11 August 2020 / Published online: 23 April 2021

(C) The Author(s) 2021

\begin{abstract}
We define a bicomplex whose Euler characteristic is the idempotented version of the ribbon element of quantum $\operatorname{sl}(2)$. We show that properties of this bicomplex descend to the centrality, invertibility, and symmetries of the ribbon element after decategorification.
\end{abstract}

Keywords Quantum $s l(2) \cdot$ Ribbon element · Bicomplex

Mathematics Subject Classification (2010) Primary 18N25 · Secondary 17B37

\section{Introduction}

The program of categorification of quantum groups was initiated by Frenkel and carried out by Khovanov and Lauda [8-10] and Rouquier [16]. In [11, 12], the 2-category $\dot{\mathcal{U}}$ was constructed that categorifies the integral idempotented version of the quantum enveloping algebra of $\mathfrak{s l}_{2}$.

The objects of $\dot{\mathcal{U}}$ are natural numbers, interpreted as the integral weight lattice of $\mathfrak{s l}_{2}$. The 1-morphisms of $\dot{\mathcal{U}}$ are generated by $\mathcal{E}^{(a)} \mathbf{1}_{n}\langle t\rangle$ and $\mathcal{F}^{(b)} \mathbf{1}_{n}\langle t\rangle$, for all $a, b \in \mathbb{N}, n, t \in \mathbb{Z}$, which are lifts of the Lusztig divided powers. The 2-morphisms are $\mathbb{Z}$-linear combinations of planar diagrams modulo local relations. The split Grothendieck group $K_{0}(\dot{\mathcal{U}})$ satisfies

$$
K_{0}\left(\mathcal{E}^{(a)} \mathbf{1}_{n}\langle t\rangle\right)=q^{t} E^{(a)} 1_{n} \quad \text { and } K_{0}\left(\mathcal{F}^{(a)} \mathbf{1}_{n}\langle t\rangle\right)=q^{t} F^{(a)} 1_{n}
$$

and coincides with the integral idempotented version of the quantum enveloping algebra $\mathbf{U}_{q}\left(\mathfrak{s l}_{2}\right)$ constructed in [1].

On the other hand, there exists a universal knot invariant [13] (see also [7]) which takes values in the center of quantum $\mathfrak{s l}_{2}$ and dominates all colored Jones polynomials. The quantum Casimir operator generating the center of $\mathbf{U}_{q}\left(\mathfrak{s l}_{2}\right)$ was categorified in [2]. This paper can be considered as the next step towards a categorification of the universal link invariant.

Anna Beliakova

anna@math.uzh.ch

Kazuo Habiro

habiro@kurims.kyoto-u.ac.jp

1 Universität Zürich, Winterthurerstr. 190, CH-8057, Zürich, Switzerland

2 Research Institute for Mathematical Sciences, Kyoto University, Kyoto, 606-8502, Japan 
The most challenging problem of this program is to find a categorical equivalent of the $R$-matrix, associated by the universal invariant to a crossing in a link diagram. Here we resolve a simpler problem, we categorify the ribbon element, which is the universal invariant of a self-crossing. The bicomplex we construct in this paper is conjecturally an element of the Drinfeld center of $\operatorname{Com}(\dot{\mathcal{U}})$ and is related to a Serre functor on category $\mathcal{O}$ associated with the longest braid [15] by Conjecture 1.3 . Here we denote by $\operatorname{Kom}(\dot{\mathcal{U}})$ the category of bicomplexes over the 2-category $\dot{\mathcal{U}}$ and by $\operatorname{Com}(\dot{\mathcal{U}})$ its homotopy version.

The ribbon element $\mathrm{r}$ is an element of the $h$-adic version $\mathbf{U}_{h}\left(\mathfrak{s l}_{2}\right)$ of the quantized enveloping algebra $\mathbf{U}_{q}\left(\mathfrak{s l}_{2}\right)$. It is given by

$$
\mathrm{r}=q^{-\frac{H^{2}}{2}-H} \sum_{k=0}^{\infty}(-1)^{k} q^{-k H-k}\left(q^{-2} ; q^{-2}\right)_{k} F^{(k)} E^{(k)},
$$

where $\left(q^{a} ; q^{b}\right)_{k}=\left(1-q^{a}\right)\left(1-q^{a+b}\right) \ldots\left(1-q^{a+(k-1) b}\right)$. Thus, the idempotented version of $r$ is

$$
\begin{aligned}
\mathrm{r} 1_{n} & =q^{-\frac{n^{2}}{2}-n} \sum_{k=0}^{\infty}(-1)^{k} q^{-k n-k}\left(q^{-2} ; q^{-2}\right)_{k} F^{(k)} E^{(k)} 1_{n} \\
& =q^{-\frac{n^{2}}{2}+n} \sum_{k=0}^{\infty}(-1)^{k} q^{k n-k}\left(q^{-2} ; q^{-2}\right)_{k} E^{(k)} F^{(k)} 1_{n}
\end{aligned}
$$

where $n \in \mathbb{Z}$.

Recall that the ribbon element is central and invertible in $\mathbf{U}_{q}\left(\mathfrak{s l}_{2}\right)$ with the inverse

$$
\mathrm{r}^{-1} 1_{n}=q^{\frac{n^{2}}{2}+n} \sum_{k=0}^{\infty}(-1)^{k} q^{k n+k}\left(q^{2} ; q^{2}\right)_{k} F^{(k)} E^{(k)} 1_{n} .
$$

The definition of the bicomplex $r \mathbf{1}_{n} \in \operatorname{Kom}(\dot{\mathcal{U}})$ categorifying the ribbon element is outlined as follows. First, we construct a bicomplex $C_{\bullet}, \bullet$ in $\operatorname{Kom}(\dot{\mathcal{U}})$

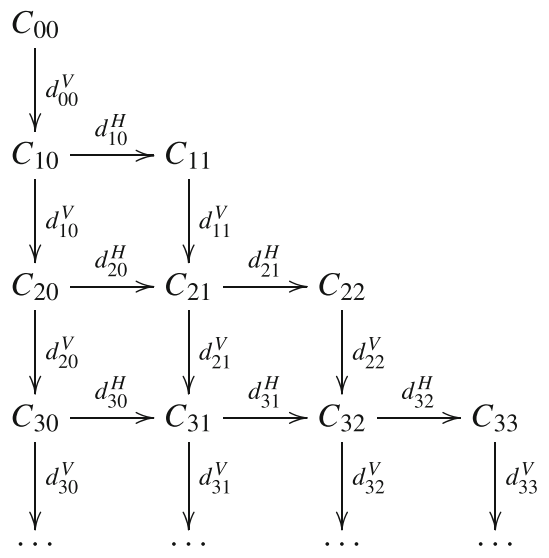

where the 1-morphism $C_{k, l}: n \rightarrow n$ in $\dot{\mathcal{U}}$ (i.e., an object $C_{k, l}$ in the additive category $\dot{\mathcal{U}}(n, n))$ is defined by

$$
C_{k, l}:=\mathcal{F}^{(k)} \mathcal{E}^{(k)} \mathbf{1}_{n}\langle-k n-k\rangle \otimes \Lambda^{l} W_{k},
$$

where $W_{k}=\operatorname{Span}_{\mathbb{Z}}\left\{w_{1}, \ldots, w_{k}\right\}$ with $\operatorname{deg}\left(w_{j}\right)=-2 j$. Note that $C_{00}=\mathcal{F}^{(0)} \mathcal{E}^{(0)} \mathbf{1}_{n}\langle 0\rangle \otimes$ $\Lambda^{0} W_{0} \cong \mathbf{1}_{n}$. The differentials $d_{k, l}^{V}, d_{k, l}^{H}$ are defined in Section 4. Figure 1 shows the 


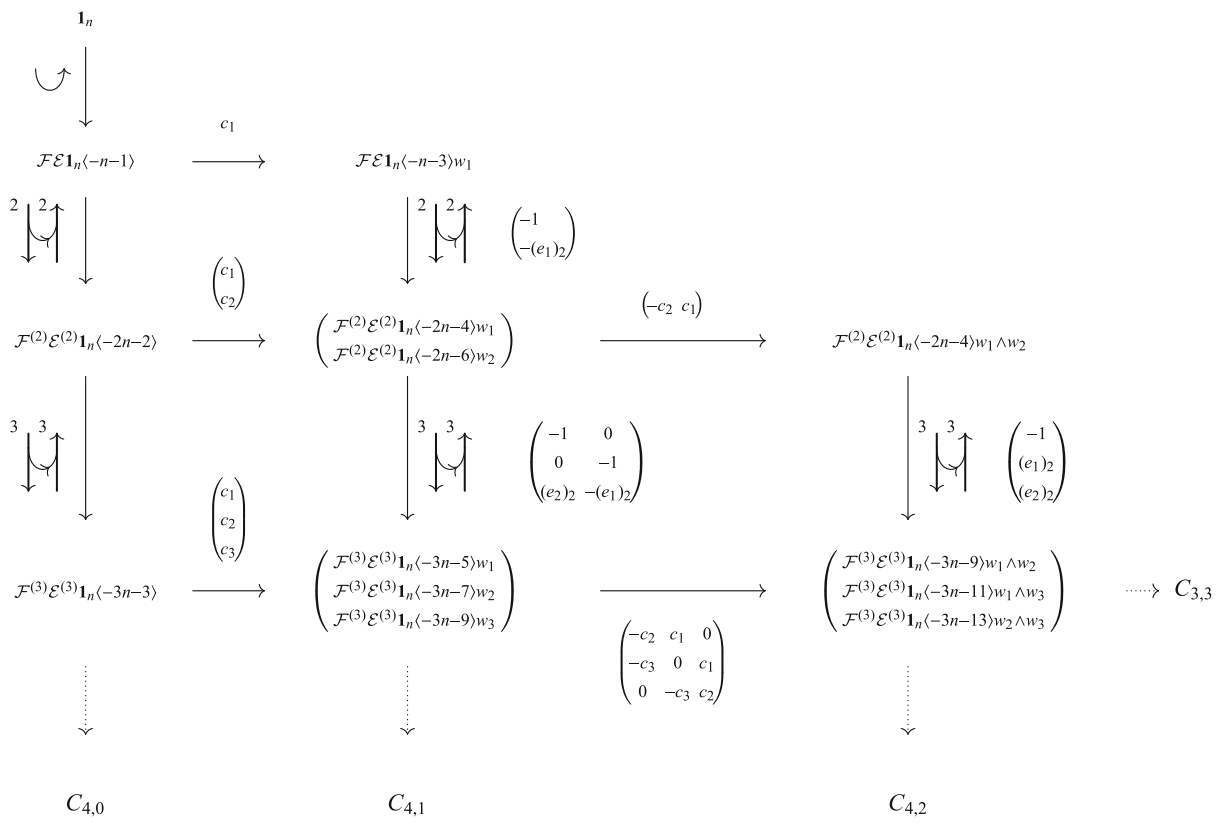

Fig. 1 The ribbon bicomplex

beginning of this bicomplex. The Euler characteristic of the bicomplex $C_{\bullet}, \bullet$ is equal to the sum part of (1.1)

$$
\sum_{k=0}^{\infty}(-1)^{k} q^{-k n-k}\left(q^{-2} ; q^{-2}\right)_{k} F^{(k)} E^{(k)} 1_{n} .
$$

Finally, we shift our bicomplex by $\left\langle-\frac{n^{2}}{2}-n\right\rangle$ in $q$-degree and by $[n / 2, n / 2]$ in homological bi-degree to obtain

$$
r \mathbf{1}_{n}:=C_{\bullet, \bullet}\left\langle-\frac{n^{2}}{2}-n\right\rangle[n / 2, n / 2] .
$$

The $q$-degree shift $\left\langle-\frac{n^{2}}{2}-n\right\rangle$ corresponds to the factor $q^{-\frac{n^{2}}{2}-n}$ in (1.1). The homological degree shift $[n / 2, n / 2]$ is added to make $r \mathbf{1}_{n}$ commute with the 1-morphisms in $\dot{\mathcal{U}}$ up to homotopy equivalence (see Theorem 1.1). We use the convention that the Euler characteristic is not affected by a global homological shift.

The bicomplex $r^{-1} \mathbf{1}_{n}$ is given by inverting all arrows in (1.2), rotating diagrams representing differentials by 180 degree, and replacing $C_{k, l}$ with ${ }^{1}$

$$
C_{k, l}^{L}:=\mathcal{F}^{(k)} \mathcal{E}^{(k)} \mathbf{1}_{n}\langle k n+k\rangle \otimes \Lambda^{l} \bar{W}_{k}, \quad \bar{W}_{k}=\operatorname{Span}_{\mathbb{Z}}\left\{\bar{w}_{1}, \ldots, \bar{w}_{k}\right\},
$$

where $\operatorname{deg}\left(\bar{w}_{j}\right)=2 j$. Finally, we have

$$
r^{-1} \mathbf{1}_{n}:=C_{\bullet, \bullet}^{L}\left\langle\frac{n^{2}}{2}+n\right\rangle[-n / 2,-n / 2] .
$$

\footnotetext{
${ }^{1}$ The index $L$ stays here for the left adjoint.
} 
It is an easy check that the Euler characteristics of $r \mathbf{1}_{n}$ and $r^{-1} \mathbf{1}_{n}$ in $\operatorname{Kom}(\dot{\mathcal{U}})$ coincide with $\mathrm{r} 1_{n}$ and $\mathrm{r}^{-1} 1_{n}$, respectively.

Let us list some properties of these bicomplexes, which follow directly from the definition.

- For each $k$, the horizontal complex $C_{k, \bullet}=\oplus_{l \in \mathbb{Z}} C_{k, l}$ forms a $k$-dimensional cube and hence is bounded.

- The total complex $\operatorname{Tot}\left(C_{\bullet, \bullet}\right)$ is a well-defined complex in $\dot{\mathcal{U}}$, i.e., for each integer $p$, $\bigoplus_{k+l=p} C_{k, l} \in \operatorname{Ob}(\dot{\mathcal{U}})$ is a finite direct sum.

- The horizontal differentials are given by certain central elements in $\operatorname{End}\left(\mathcal{F}^{(k)} \mathcal{E}^{(k)} \mathbf{1}_{n}\right)$ defined in Section 3 and generated by dots.

Recall that $\dot{\mathcal{U}}$ is the Karoubi envelope of the 2-category $\mathcal{U}$ defined in [12]. The generators for 1-morphisms in $\mathcal{U}$ are $\mathcal{E} \mathbf{1}_{n}\langle t\rangle$ and $\mathcal{F} \mathbf{1}_{n}\langle t\rangle$ for $n, t \in \mathbb{Z}$. The involutive 2-functors $\omega, \sigma$ and $\psi$, generating the symmetry group $\mathcal{G}=(\mathbb{Z} / 2 \mathbb{Z})^{3}$ of $\mathcal{U}$ were also introduced in [12] and [2]. We recall these definitions and extend them to $\dot{\mathcal{U}}$ in Section 10. Let us denote by $\mathcal{G}_{1}:=\{1, \sigma \omega\}$ the subgroup of $\mathcal{G}$.

Throughout this paper, we adopt the notation $r r^{-1} \mathbf{1}_{n}$ for the tensor product of two complexes, i.e., we omit tensor sign, since on 1-morphisms of $\dot{\mathcal{U}}$ tensor product is just a concatenation.

Our main result is the following:

Theorem 1.1 (Centrality) For any complex $X \in \operatorname{Com}(\mathcal{U})$, there are isomorphisms

$$
\kappa_{X}: X r \mathbf{1}_{n} \rightarrow r X \mathbf{1}_{n}, \quad \eta_{X}: X r^{-1} \mathbf{1}_{n} \rightarrow r^{-1} X \mathbf{1}_{n}
$$

of bicomplexes in $\operatorname{Com}(\dot{\mathcal{U}})$.

(Invertibility) The bicomplexes $r r^{-1} \mathbf{1}_{n}$ and $r^{-1} r \mathbf{1}_{n}$ in ${ }^{l} \operatorname{Kom}(\dot{\mathcal{U}})$ are homotopy equivalent to $\mathbf{1}_{n}$.

(Symmetry) The bicomplexes $r \mathbf{1}_{n}$ and $r^{-1} \mathbf{1}_{n}$ in $\operatorname{Kom}(\dot{\mathcal{U}})$ are invariant under the action of $\mathcal{G}_{1} \subset \mathcal{G}$. Moreover, $r^{-1} \mathbf{1}_{n}=\sigma \omega \psi\left(r \mathbf{1}_{n}\right)$ is isomorphic to $\psi\left(r \mathbf{1}_{n}\right)$.

Note that since the bicomplexes $r \mathbf{1}_{n}$ and $r^{-1} \mathbf{1}_{n}$ are bounded from above and below respectively, and $\dot{\mathcal{U}}$ does not admit infinite direct sums, their tensor product $r r^{-1} \mathbf{1}_{n}$ does not belong to $\operatorname{Kom}(\dot{\mathcal{U}})$. Instead we are using ${ }^{l} \operatorname{Kom}(\dot{\mathcal{U}})$ which is the inverse limit of the categories of bounded bicomplexes $\operatorname{Kom}^{b}\left(\dot{\mathcal{U}}_{N}\right)$, where $\dot{\mathcal{U}}_{N}$ is the Schur quotient of $\dot{\mathcal{U}}$ defined by setting $\mathbf{1}_{N+2}=0$ (see Section 12 for more details).

Properties listed in Theorem 1.1 are natural lifts of the properties of the ribbon element to higher categorical level. We would also expect the following to hold.

Conjecture 1.2 (Naturality) The chain maps $\kappa_{X}$ and $\eta_{X}$ are natural, i.e., they commute with all 2-morphisms of $\dot{\mathcal{U}}$.

(Decomposability) For $n \geq 0$ the bicomplex $r \mathbf{1}_{n}$ is indecomposable in $\operatorname{Kom}(\dot{\mathcal{U}})$, and the bicomplex $\omega\left(r \mathbf{1}_{-n}\right)$ is isomorphic to a direct sum of $r \mathbf{1}_{n}$ and a contractible complex. For $n \leq 0$ the bicomplex $\omega\left(r \mathbf{1}_{-n}\right)$ is indecomposable in $\operatorname{Kom}(\dot{\mathcal{U}})$, and the bicomplex $r \mathbf{1}_{n}$ is isomorphic to a direct sum of $\omega\left(r \mathbf{1}_{-n}\right)$ and a contractible complex. For $n=0$, the bicomplexes $r \mathbf{1}_{n}$ and $\omega\left(r \mathbf{1}_{-n}\right)$ are isomorphic.

Observe that we could use $\sigma\left(r \mathbf{1}_{-n}\right)$ instead of $\omega\left(r \mathbf{1}_{-n}\right)$, since by Theorem 1.1 they are isomorphic. 
Let us comment on this conjecture. We expect the isomorphisms $\kappa_{X}$ and $\eta_{X}$ to be natural, and hence, to be defined for any $X \in \operatorname{Com}(\dot{\mathcal{U}})$. This would imply that the bicomplexes $r \mathbf{1}_{n}$ and $r^{-1} \mathbf{1}_{n}$ belong to the Drinfeld center of $\operatorname{Com}(\dot{\mathcal{U}})$ viewed as an additive monoidal category. Here we regard 1-morphisms in $\operatorname{Com}(\dot{\mathcal{U}})$ as objects of the monoidal category. The monoidal structure is given by composition of 1-morphisms and horizontal composition of 2-morphisms. The collection of chain maps $\kappa_{X}$ define then an invertible natural transformation $\kappa:-r \Longrightarrow r$ - between endofunctors of $\operatorname{Com}(\dot{\mathcal{U}})$ given by tensoring on the left and on the right with the complex $r \mathbf{1}_{n}$ for an appropriate $n$.

The first column of our bicomplex $C_{00} \rightarrow C_{10} \rightarrow C_{20} \rightarrow \ldots$ is an example of socalled Rickard complex introduced by Chuang and Rouquier in [6] and intensively studied by Cautis and Kamnitzer [4, 5]. The Rickard-Rouquier complex $\mathbf{1}_{-n} \mathbf{T} \mathbf{1}_{n}$ categorifies the action of the Weyl group on the finite-dimensional representations and satisfies the braid relation. It can be defined as follows:

$$
\begin{array}{cr}
\mathrm{T} \mathbf{1}_{n}: \ldots \rightarrow \mathcal{F}^{(n+s)} \mathcal{E}^{(s)}\langle s\rangle \mathbf{1}_{n} \rightarrow \mathcal{F}^{(n+s-1)} \mathcal{E}^{(s-1)}\langle s-1\rangle \mathbf{1}_{n} \rightarrow \ldots \rightarrow \mathcal{F}^{(n)} \mathbf{1}_{n} & \text { for } n \geq 0, \\
\mathrm{~T} \mathbf{1}_{n}: \ldots \rightarrow \mathcal{E}^{(-n+s)} \mathcal{F}^{(s)}\langle s\rangle \mathbf{1}_{n} \rightarrow \mathcal{E}^{(-n+s-1)} \mathcal{F}^{(s-1)}\langle s-1\rangle \mathbf{1}_{n} \rightarrow \ldots \rightarrow \mathcal{E}^{(-n)} \mathbf{1}_{n} & \text { for } n \leq 0,
\end{array}
$$

where the differential are non-zero maps.

Conjecture 1.3 The total complex of the ribbon bicomplex $\operatorname{Tot}\left(r^{-1} \mathbf{1}_{n}\right)$ is homotopy equivalent to

$$
\mathrm{T}^{2} \mathbf{1}_{n}\left\langle\frac{n^{2}}{2}+n\right\rangle[-n / 2,-n / 2] .
$$

Note that the decategorified version of this conjecture holds. For $n \geq 0$, the Euler characteristic

$$
\begin{aligned}
T^{2} 1_{n}=T 1_{-n} T 1_{n} & =\sum_{l, s \geq 0}(-q)^{l+s} E^{(n+s)} F^{(s)} F^{(n+l)} E^{(l)} 1_{n} \\
& =\sum_{k=0}^{\infty}(-1)^{k} q^{k n+k}\left(q^{2} ; q^{2}\right)_{k} F^{(k)} E^{(k)} 1_{n}
\end{aligned}
$$

coincides with $\mathrm{r}^{-1} 1_{n}$ after multiplying with $q^{n^{2} / 2+n}$. The case $n \leq 0$ can be obtained similarly, after replacing $n$ by $-n$ and exchanging $E$ 's and $F$ 's.

\subsection{Strategy of the Proof of Theorem 1.1}

It is enough to check centrality on the generators. This is because any "chain group" of $X$ is a composition of $\mathcal{E}$ 's and $\mathcal{F}$ 's and the maps $\eta_{\mathcal{E}}$ and $\eta_{\mathcal{F}}$ are adjoint to $\kappa_{\mathcal{F}}$ and $\kappa_{\mathcal{E}}$, respectively.

Lemma 1.4 There are maps

$$
\kappa_{\mathcal{E}}: \mathcal{E} r \mathbf{1}_{n} \rightarrow r \mathcal{E} \mathbf{1}_{n}, \quad \bar{\kappa}_{\mathcal{F}}: r \mathcal{F} \mathbf{1}_{n+2} \rightarrow \mathcal{F} r \mathbf{1}_{n+2}
$$

which are homotopy equivalences in $\operatorname{Kom}(\dot{\mathcal{U}})$.

Here $r \mathcal{E} \mathbf{1}_{n}$ is the bicomplex obtained by composing $r \mathbf{1}_{n+2}$ to the left of $\mathcal{E} \mathbf{1}_{n}$. The differentials are those of $r \mathbf{1}_{n+2}$ extended by identity on $\mathcal{E} \mathbf{1}_{n}$. The other bicomplexes are defined analogously. 
To construct $\kappa_{\mathcal{E}}$, we will proceed as follows. We will define an intermediate bicomplex $\left(r \mathcal{E} \mathbf{1}_{n}\right)^{\prime}$ as an indecomposable summand of $r \mathcal{E} \mathbf{1}_{n}$, whose "chain groups" are

$$
C_{k, l}^{\prime}=\mathcal{F}^{(k)} \mathcal{E}^{(k+1)} \mathbf{1}_{n}\langle-k n-2 k\rangle \otimes \Lambda^{l} W_{k}^{\prime}
$$

with $W_{k}^{\prime}:=\operatorname{Span}_{\mathbb{Z}}\left\{w_{2}, \ldots, w_{k+1}\right\}$, the total $q$-degree shift $\left\langle-\frac{n^{2}}{2}-3 n-4\right\rangle$ and the homological shift $[n / 2+1, n / 2+1]$. Then we show that $\mathcal{E} r \mathbf{1}_{n}$ and $r \mathcal{E} \mathbf{1}_{n}$ retract to $\left(r \mathcal{E} \mathbf{1}_{n}\right)^{\prime}$ (Theorems 7.1, 5.3). Composing the corresponding homotopy equivalences we will get $\kappa_{\mathcal{E}}$. The construction of the chain maps between $\mathcal{E} r \mathbf{1}_{n}, r \mathcal{E} \mathbf{1}_{n}$ and $\left(r \mathcal{E} \mathbf{1}_{n}\right)^{\prime}$, and the proofs of Theorems 7.1 and 5.3 are the most involved technical part of the paper.

To define $\kappa_{\mathcal{F}}$, we use the invariance of $r \mathbf{1}_{n}$ under $\sigma \omega$. Indeed, we have

$$
\sigma \omega(\kappa \mathcal{E}): \sigma \omega\left(\mathcal{E} r \mathbf{1}_{n}\right) \rightarrow \sigma \omega\left(r \mathcal{E} \mathbf{1}_{n}\right) .
$$

However, $\sigma \omega\left(\mathcal{E} r \mathbf{1}_{n}\right)=\sigma \omega(r) \mathcal{F} \mathbf{1}_{n+2} \simeq r \mathcal{F} \mathbf{1}_{n+2}$ and similarly, $\sigma \omega\left(r \mathcal{E} \mathbf{1}_{n}\right)$ is isomorphic to $\mathcal{F} r \mathbf{1}_{n+2}$.

The proof of invertibility is based on the next theorem computing the action of the ribbon complex on the category of complexes over Flag $_{N}$ defined in [12] and the main result of [3]. Let us define the endofunctors $r_{N}^{L}$ and $r_{N}^{R}$ of $\operatorname{Com}\left(\mathbf{F l a g}_{N}\right)$ by tensoring with $\Gamma_{N}\left(r \mathbf{1}_{n}\right)$ on the left and right, respectively, i.e., $r_{N}^{L}(X)=\Gamma_{N}\left(r \mathbf{1}_{n}\right) X$. Analogously, the endofunctors $\left(r^{-1}\right)_{N}^{L}$ and $\left(r^{-1}\right)_{N}^{R}$ are defined by tensoring with $\Gamma_{N}\left(r^{-1} \mathbf{1}_{n}\right)$.

Theorem 1.5 For any natural number $N, r_{N}^{L}$ and $r_{N}^{R}$ are the identity endofunctors of $\operatorname{Com}\left(\mathbf{F l a g}_{N}\right)$ up to degree shift. Their inverses are $\left(r^{-1}\right)_{N}^{L}$ and $\left(r^{-1}\right)_{N}^{R}$, respectively.

The main result of [3] says that $\dot{\mathcal{U}}$ is the inverse limit of Flag 2-categories. Hence, we conclude that tensoring with $r^{ \pm 1} r^{\mp 1} \mathbf{1}_{n}$ is the inverse limit of the identity functor in $\mathbf{F l a g}_{N}$, which is the identity endofunctor of ${ }^{l} \operatorname{Com}(\dot{\mathcal{U}})$.

To prove symmetry, we construct a bicomplex $\tilde{r} \mathbf{1}_{n}$ which is (1) isomorphic to $r \mathbf{1}_{n}$ in $\operatorname{Kom}(\dot{\mathcal{U}})$ and $(2)$ invariant under $\sigma \omega$. This is done in Section 11. Then, given the isomorphism $H: r \mathbf{1}_{n} \rightarrow \tilde{r} \mathbf{1}_{n}$, the composition $\sigma \omega\left(H^{-1}\right) \circ H: r \mathbf{1}_{n} \rightarrow \sigma \omega\left(r \mathbf{1}_{n}\right)$ is the required isomorphism.

The paper is organized as follows. After some preliminaries, we define the central elements $c_{\lambda}$ indexed by partitions, $r \mathbf{1}_{n}, r^{-1} \mathbf{1}_{n}$ and the intermediate bicomplex $\left(r \mathcal{E} \mathbf{1}_{n}\right)^{\prime}$. The next four sections are devoted to the definition of the chain maps and homotopies and to the proofs of Theorems 7.1, 5.3 and Theorem 1.1 (Centrality). After that we recall the definitions of the symmetry 2-functors and define the images of $r \mathbf{1}_{n}$ under those symmetries. Section 11 is devoted to the construction of $\tilde{r} \mathbf{1}_{n}$. In the last section, we prove Theorem 1.5 and Theorem 1.1 (Invertibility).

In Appendix, we collect identities needed for the proofs.

\section{General Facts}

\subsection{Definitions and Conventions}

We refer to [12] and [11] for the definitions of the 2-categories $\mathcal{U}$ and its Karoubi envelope $\dot{\mathcal{U}}$. The 2-morphisms in these 2-categories are given by diagrams modulo some local relations. The right most region in all our diagrams is labeled by $n$. For any 1-morphism $x \in \operatorname{Hom}_{\dot{\mathcal{U}}}(n, m)$, we denote by $\operatorname{Dot}(x) \subset \operatorname{End}_{\dot{\mathcal{U}}}(x)$ the subspace of its 2-endomorphisms generated by dots. A thick line labeled with a positive integer $k$ denotes the identity 
2-morphism of $\left.\mathcal{E}^{(k)}:=\left(\mathcal{E}^{k},\right\rangle_{k}\right)\left\langle-\frac{k(k-1)}{2}\right\rangle$ if it is oriented upwards; and the identity 2morphism of $\left.\mathcal{F}^{(k)}:=\left(\mathcal{F}^{k},\right\rangle_{k}^{\prime}\right)\left\langle\frac{k(k-1)}{2}\right\rangle$ in $\dot{\mathcal{U}}$ otherwise, where $\rangle_{k}$ is the idempotent defined in [11] and $\rangle_{k}^{\prime}$ is its image under 180-degree rotation. The case $k=1$ will be represented by a thin line without any label for a better visibility.

In this paper, for any 2 -category $\mathcal{C}$, we denote by $\operatorname{Kom}(\mathcal{C})$ the 2-category of bicomplexes over the 2 -category $\mathcal{C}$. The objects of $\operatorname{Kom}(\mathcal{C})$ coincide with objects of $\mathcal{C}, 1$-morphisms are bicomplexes of 1-morphisms in $\mathcal{C}$, and 2-morphisms are chain maps, constructed from 2morphisms in $\mathcal{C}$. Let $\operatorname{Com}(\mathcal{C})$ be the 2-category with the same objects and 1-morphisms as $\operatorname{Kom}(\mathcal{C})$ but whose 2-morphisms are chain maps up to homotopy. We will denote by $\operatorname{Kom}^{b}(\mathcal{C})$ and $\operatorname{Com}^{b}(\mathcal{C})$ corresponding bounded versions.

The degree of a 2-morphism in $\mathcal{U}$ is defined as degree of the target minus degree of the source plus degree of the diagram.

\subsection{Strong Deformation Retraction}

Let us recall the definitions.

A chain complex $\left(C^{\prime}, d^{\prime}\right)$ is a strong deformation retract of a chain complex $(C, d)$ if there exist

- A chain map $f: C \rightarrow C^{\prime}$, i.e., $d^{\prime} f=f d$;

- A chain map $g: C^{\prime} \rightarrow C$, i.e., $d g=g d^{\prime}$;

- A homotopy $h: C_{\bullet} \rightarrow C_{\bullet-1}$ satisfying $h d+d h=1-g f$ and

$$
\begin{array}{rlrl}
f g & =1 & h^{2} & =0 \\
f h & =0 & h g & =0 .
\end{array}
$$

From the above four equalities including $g$, it is enough to show that $f g=1$ and $h d+$ $d h=1-g f$. The other two equalities $\left(d g=g d^{\prime}, h g=0\right)$ follow from them.

Let $\left(C, d^{V}, d^{H}\right)$ and $\left(C^{\prime}, d^{\prime V}, d^{\prime H}\right)$ be two bicomplexes. We say that the second bicomplex is a strong deformation retract of the first one if there exist

- A chain map $f: C \rightarrow C^{\prime}$ with $d^{\prime H} f=f d^{H}, d^{\prime V} f=f d^{V}$;

- A chain map $g: C^{\prime} \rightarrow C$ with $d^{H} g=g d^{\prime H}, d^{V} g=g d^{\prime V}$;

- A homotopy $h=h^{H}+h^{V}: C_{\bullet} \rightarrow C_{\bullet-1}$ with $h^{H} d^{H}+h^{V} d^{V}+d^{H} h^{H}+d^{V} h^{V}=$ $1-g f, h^{H} d^{V}+d^{V} h^{H}=0$ and $h^{V} d^{H}+d^{H} h^{V}=0$ satisfying

$$
\begin{array}{cccc}
f g=1 & h^{H} h^{H}=0 & h^{H} h^{V}+h^{V} h^{H}=0 & h^{V} h^{V}=0 \\
f h^{H}=0 & f h^{V}=0 & h^{H} g=0 & h^{V} g=0 .
\end{array}
$$

Remark The equalities $d^{H} g=g d^{\prime H}, d^{V} g=g d^{\prime V}, h^{H} g=0$ and $h^{V} g=0$ follow from the others.

\subsection{Symmetric Functions}

Let us denote by $S_{k}$ the symmetric group and $A_{k}=\mathbb{Z}\left[x_{1}, \ldots, x_{k}\right]^{S_{k}}$ the ring of symmetric polynomials. Let $A$ be the ring of symmetric functions, defined as the inverse limit of the system $\left(A_{k}\right)_{k \in \mathbb{N}}$.

For a partition $\lambda=\left(\lambda_{1}, \lambda_{2}, \ldots, \lambda_{a}\right)$ with $\lambda_{1} \geq \lambda_{2} \geq \cdots \geq \lambda_{a} \geq 0$ let $|\lambda|:=\sum_{i=1}^{a} \lambda_{i}$. We denote by $P(a)$ the set of all partitions $\lambda$ with at most $a$ parts (i.e., with $\lambda_{a+1}=0$ ). Moreover, the set of all partitions (i.e., the set $P(\infty)$ ) we denote simply by $P$.

The dual (conjugate) partition of $\lambda$ is the partition $\lambda^{t}=\left(\lambda_{1}^{t}, \lambda_{2}^{t}, \ldots\right)$ with $\lambda_{j}^{t}=\sharp\left\{i \mid \lambda_{i} \geq\right.$ $j$ \} which is given by reflecting the Young diagram of $\lambda$ along the diagonal. 
The Schur polynomials $\left\{s_{\lambda} \mid \lambda \in P(k)\right\}$ form a basis of $A_{k}$, as well as Schur functions $\left\{s_{\lambda} \mid \lambda \in P\right\}$ is a base of $A$. The multiplication in this basis is given by the following formula

$$
s_{\mu} s_{\nu}=\sum_{\lambda \in P} N_{\mu \nu}^{\lambda} s_{\lambda}
$$

where $N_{\mu \nu}^{\lambda}$ are the Littlewood-Richardson coefficients. The elementary symmetric functions $\left\{s_{1^{d}}=e_{d} \mid d \in \mathbb{N}\right\}$ or the complete symmetric functions $\left\{s_{d}=h_{d} \mid d \in \mathbb{N}\right\}$ generate $A$ multiplicatively.

The ring $A$ has a natural Hopf algebra structure with comultiplication

$$
\Delta: A \longrightarrow A \otimes A
$$

given by

$$
\Delta\left(s_{\lambda}\right)=\sum_{\mu, \nu \in P} N_{\mu \nu}^{\lambda} s_{\mu} \otimes s_{\nu},
$$

and with counit and antipode defined as follows

$$
\begin{aligned}
& \epsilon: A \longrightarrow \mathbb{Z} \quad \gamma: A \longrightarrow A \\
& s_{\lambda} \mapsto \delta_{\lambda, 0}, \quad s_{\lambda} \mapsto(-1)^{|\lambda|} s_{\lambda^{t}} .
\end{aligned}
$$

\section{The Center of the 2-category $\dot{\mathcal{U}}$}

After recalling the general definition of a center for any linear category, we construct central elements in $\dot{\mathcal{U}}(n, m)$.

\subsection{Center of a Category}

For a linear category $C$, the center $Z(C)$ of $C$ is the ring of endo-natural transformations on the identity functor $1_{C}: C \longrightarrow C$. Thus, an element $\sigma$ of $Z(C)$ is a collection of endomorphisms $\sigma_{x}: x \longrightarrow x$ for objects $x$ in $C$ such that we have

$$
f \sigma_{x}=\sigma_{y} f
$$

for any morphisms $f: x \longrightarrow y$ in $C$. Multiplication of two elements $\sigma$ and $\tau$ in $Z(C)$ is defined by

$$
(\tau \sigma)_{x}:=\tau_{x} \sigma_{x}
$$

It is easily seen that $Z(C)$ is commutative. We call $\sigma$ a central element of $C$.

Let $\mathcal{C}$ be a linear 2-category. For each pair $(x, y)$ of objects in $\mathcal{C}$, one can consider the center $Z(\mathcal{C}(x, y))$ of the category $\mathcal{C}(x, y)$ whose objects are the 1-morphisms between $x$ and $y$ and morphisms are 2-morphisms. The center $Z(\mathcal{C}(x, y))$ is a commutative ring.

\subsection{Central Elements in $\dot{\mathcal{U}}$}

We have natural ring homomorphisms

$$
\begin{aligned}
& d_{a}: A_{a} \longrightarrow \operatorname{Dot}\left(\mathcal{E}^{(a)} \mathbf{1}_{n}\right), \\
& d_{a}^{\prime}: A_{a} \longrightarrow \operatorname{Dot}\left(\mathcal{F}^{(a)} \mathbf{1}_{n}\right)
\end{aligned}
$$


that lift to

$$
\begin{aligned}
& d_{a}: A \longrightarrow \operatorname{Dot}\left(\mathcal{E}^{(a)} \mathbf{1}_{n}\right), \\
& d_{a}^{\prime}: A \longrightarrow \operatorname{Dot}\left(\mathcal{F}^{(a)} \mathbf{1}_{n}\right)
\end{aligned}
$$

by sending elementary symmetric functions of degree higher than $a$ to zero.

In this subsection, for each 1-morphism $f: n \longrightarrow m$ in $\dot{\mathcal{U}}$, we define a ring homomorphism

$$
c=c_{f}: A \longrightarrow \operatorname{Dot}(f)
$$

For $f=\mathcal{E}^{(a)} \mathbf{1}_{n}$, we set

$$
c_{\mathcal{E}^{(a)} \mathbf{1}_{n}}(x)=d_{a}(x)
$$

For $f=\mathcal{F}^{(a)} \mathbf{1}_{n}$, we set

$$
c_{\mathcal{F}^{(a)} \mathbf{1}_{n}}(x)=d_{a}^{\prime}(\gamma(x)) .
$$

For $f=f_{1} f_{2} \ldots f_{p}$, where each $f_{j}$ is $\mathcal{E}^{\left(a_{j}\right)} \mathbf{1}_{n_{j}}$ or $\mathcal{F}^{\left(a_{j}\right)} \mathbf{1}_{n_{j}}, a_{j} \geq 0, n_{j} \in \mathbb{Z}$, we set

$$
\begin{aligned}
c_{f_{1} f_{2} \ldots f_{p}}(x) & =\text { horizontal composition }\left(\left(c_{f_{1}} \otimes \cdots \otimes c_{f_{p}}\right) \Delta^{[p]}(x)\right) \\
& =\sum c_{f_{1}}\left(x_{(1)}\right) \circ \cdots \circ c_{f_{p}}\left(x_{(p)}\right),
\end{aligned}
$$

where $\Delta^{[p]}: A \longrightarrow A^{\otimes p}$ is the $p$-output comultiplication with

$$
\Delta^{[p]}(x)=\sum x_{(1)} \otimes \cdots \otimes x_{(p)} .
$$

Finally, for a direct sum $f=f_{1} \oplus \cdots \oplus f_{p}$, we set

$$
c_{f_{1} \oplus \cdots \oplus f_{p}}(x)=\operatorname{diag}\left(c_{f_{1}}(x), \ldots, c_{f_{p}}(x)\right) .
$$

We also adopt the notation $\left(c_{\lambda}\right)_{f}$ for $c_{f}\left(s_{\lambda}\right)$ and draw

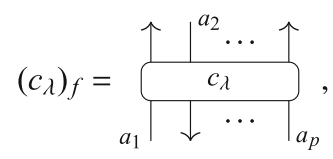

where $f=f_{1} f_{2} \ldots f_{p}$, each $f_{j}$ is $\mathcal{E}^{\left(a_{j}\right)} \mathbf{1}_{n_{j}}$ or $\mathcal{F}^{\left(a_{j}\right)} \mathbf{1}_{n_{j}}$.

For $f=\mathcal{E}^{a} \mathbf{1}_{n}$ and $f=\mathcal{F}^{a} \mathbf{1}_{n}$, we have

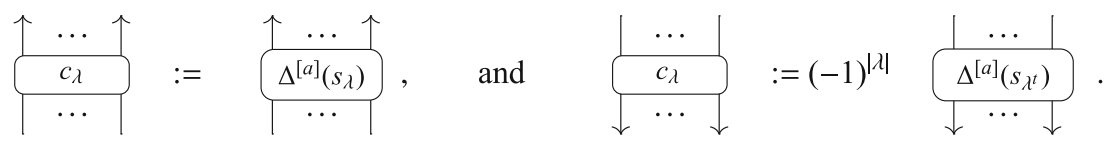

For $f=\mathcal{E}^{(a)} \mathbf{1}_{n}$ and $f=\mathcal{F}^{(a)} \mathbf{1}_{n}$, we have

$$
\overbrace{c_{a}}^{c_{\lambda}}=\uparrow_{a}^{\lambda}, \quad \underbrace{c_{\lambda}}_{\perp}=\left.(-1)^{|\lambda|}\right|^{a} .
$$

The following proposition is the direct consequence of the definitions.

Proposition 3.1 For 1-morphisms $f: n \longrightarrow l$ and $g: l \longrightarrow m$ in $\dot{\mathcal{U}}$, we have

$$
\left(c_{\lambda}\right)_{g f}=\sum_{\mu, \nu \in P} N_{\mu, v}^{\lambda}\left(c_{\mu}\right)_{g} \circ\left(c_{\nu}\right)_{f}
$$


and

$$
\left(c_{\lambda} c_{\mu}\right)_{g f}=\sum_{\nu \in P} N_{\lambda, \mu}^{v}\left(c_{\nu}\right)_{g f}
$$

In particular, for $\lambda \in P_{d}$, and $f=\mathcal{E}^{\left(i_{1}\right)} \mathcal{F}^{\left(j_{1}\right)} \ldots \mathcal{E}^{\left(i_{p}\right)} \mathcal{F}^{\left(j_{p}\right)} \mathbf{1}_{n}$, we have

$$
=\sum_{\lambda^{(1)}, \mu^{(1)}, \ldots, \lambda^{(p)}, \mu^{(p)} \in P}^{\left(c_{\lambda}\right)_{\mathcal{E}^{\left(i_{1}\right)} \mathcal{F}^{\left(j_{1}\right)} \ldots \mathcal{E}^{(i p)} \mathcal{F}^{(j p)} \mathbf{1}_{n}}(-1)^{l_{1}+\cdots+l_{p}} N_{\lambda^{(1)} \mu^{(1)} \ldots \lambda^{(p)} \mu^{(p)}}^{\lambda} \lambda^{(1)} \circ\left(\mu^{(1)}\right)^{t} \circ \cdots \circ \lambda^{(p)} \circ\left(\mu^{(p)}\right)^{t},}
$$

where $N_{\lambda^{(1)} \mu^{(1)} \ldots \lambda^{(p)} \mu^{(p)}}^{\lambda} \in \mathbb{Z}_{\geq 0}$ are the Littlewood-Richardson coefficients.

\section{Examples}

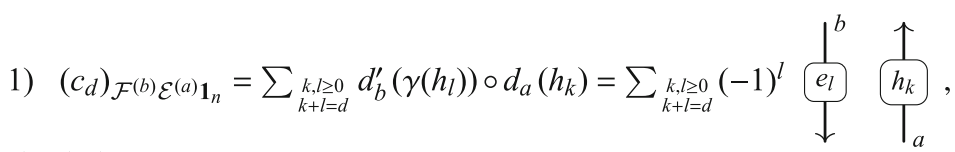

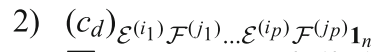

$$
\begin{aligned}
& =\sum_{\substack{k_{1}, l_{1}, \ldots, k_{p}, l_{p} \geq 0 \\
k_{1}+l_{1}+\cdots+k_{p}+l_{p}=d}} d_{i_{1}}\left(h_{k_{1}}\right) \circ d_{j_{1}}^{\prime}\left(\gamma\left(h_{l_{1}}\right)\right) \circ \cdots \circ d_{i_{p}}\left(h_{k_{p}}\right) \circ d_{j_{p}}^{\prime}\left(\gamma\left(h_{l_{p}}\right)\right)
\end{aligned}
$$

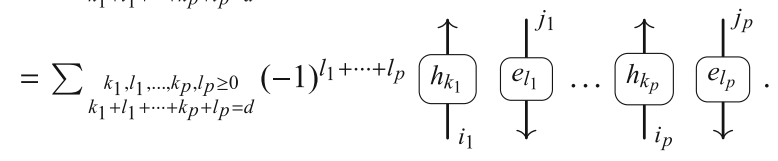

Proposition 3.2 For $m, n \in \mathbb{Z}, \lambda \in P$ we have $c_{\lambda} \in Z(\dot{\mathcal{U}}(n, m))$.

Proof We need to prove that for every 2-morphism $\alpha: f \rightarrow g$ in $\dot{\mathcal{U}}(n, m)$ we have

$$
\alpha\left(c_{\lambda}\right)_{f}=\left(c_{\lambda}\right)_{g} \alpha .
$$

Splitting the thick lines and moving the dots as follows
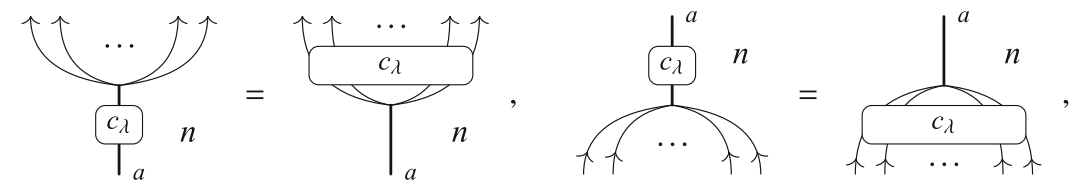

we see that it is enough to prove the proposition for 2-morphisms of $\mathcal{U}$, generated by dots, crossings and turns. For dots, the proposition is clear.

Since elementary functions generate $A$, it is enough to consider $c_{\lambda}$ for $\lambda=1^{d}, d \in \mathbb{N}$ in what follows. Note that $\left(c_{1^{d}}\right)_{\mathcal{E}^{2} \mathbf{1}_{n}} \neq 0$ only for $d=1$ or $d=2$ and

$$
\uparrow \uparrow+\uparrow+\uparrow, \quad\left\{\begin{array}{c}
\uparrow c_{(1,1)} \\
c_{(1)}
\end{array}=\uparrow \uparrow .\right.
$$

Using the NilHecke relations, we can easily check that both upper 2-morphisms commute with the crossing. The same is true for downward oriented arrows.

Similarly, we have

$$
\downarrow c_{1^{d}} \uparrow=\iota^{d} \uparrow-\bullet^{d-1} \uparrow .
$$


We see that by multiplying with the turn from below, we get 0 , which coincides with $\left(c_{1^{d}}\right)_{1_{n}}=0$. The other turns can be proved similarly.

\section{Definitions of $r 1_{n}, r^{-1} 1_{n}$ and $\left(r E 1_{n}\right)^{\prime}$}

\subsection{Ribbon Bicomplex}

As it was already mentioned in Section 1, the bicomplex $r \mathbf{1}_{n}$ categorifying the ribbon element has "chain groups"

$$
C_{k, l}:=\mathcal{F}^{(k)} \mathcal{E}^{(k)} \mathbf{1}_{n}\langle-k n-k\rangle \otimes \Lambda^{l} W_{k}, \quad W_{k}=\operatorname{Span}_{\mathbb{Z}}\left\{w_{1}, \ldots, w_{k}\right\}, \quad \operatorname{deg}\left(w_{j}\right)=-2 j
$$

with the total $q$-degree shift $\left\langle-\frac{n^{2}}{2}-n\right\rangle$ and homological shift $[n / 2, n / 2]$. Here note that for an additive category $\mathcal{C}$ and a free abelian group $G$ of finite rank, one can construct a functor $-\otimes G: \mathcal{C} \rightarrow \mathcal{C}$.

The horizontal differential $d_{k, l}^{H}: C_{k, l} \rightarrow C_{k, l+1}$ sends $x \mapsto c \wedge x$, where

$$
c:=\sum_{j=1}^{k} c_{j} \otimes w_{j}:=\sum_{j=1}^{k}(\sum_{i=0}^{j}(-1)^{i} \underbrace{e_{i}^{k}}_{\downarrow} \overbrace{h_{j-i}}^{\uparrow^{k}}) \otimes w_{j} \in \operatorname{Dot}\left(\mathcal{F}^{(k)} \mathcal{E}^{(k)} \mathbf{1}_{n}\right) \otimes W_{k}
$$

are our central elements.

To define the vertical differential, we proceed as follows. Consider the linear map

$$
\begin{aligned}
\alpha_{k}: W_{k} & \rightarrow \operatorname{Dot}\left(\mathcal{E}^{(k)} \mathbf{1}_{n}\right) \otimes W_{k+1} \\
\alpha_{k}\left(w_{i}\right) & =\mathbf{1} \otimes w_{i}-(-1)^{k+1-i} \stackrel{\uparrow^{k}}{e_{k+1-i}} \otimes w_{k+1},
\end{aligned}
$$

where 1 denotes the identity 2-morphism of $\mathcal{E}^{(k)} 1_{n}$. This map induces an algebra homomorphism

$\alpha_{k}: \Lambda^{\bullet} W_{k} \rightarrow \operatorname{Dot}\left(\mathcal{E}^{(k)} \mathbf{1}_{n}\right) \otimes \Lambda^{\bullet} W_{k+1} \quad$ and let $\quad \alpha_{k, l}: \Lambda^{l} W_{k} \rightarrow \operatorname{Dot}\left(\mathcal{E}^{(k)} \mathbf{1}_{n}\right) \otimes \Lambda^{l} W_{k+1}$ be its degree $l$ part. Then the vertical differential is

$$
\begin{aligned}
& d_{k, l}^{V}:=(-1)^{l} \overbrace{\underbrace{\alpha_{k, l}}_{k}}^{\overbrace{\alpha_{k}}^{k+1}}: C_{k, l} \rightarrow C_{k+1, l} \text {, where } \\
& \alpha_{k, l}\left(w_{i_{1}} \wedge w_{i_{2}} \wedge \cdots \wedge w_{i_{l}}\right)=\alpha_{k}\left(w_{i_{1}}\right) \wedge \alpha_{k}\left(w_{i_{2}}\right) \wedge \cdots \wedge \alpha_{k}\left(w_{i_{l}}\right) \\
& =\mathbf{1} \otimes w_{i_{1}} \wedge \cdots \wedge w_{i_{l}}-\sum_{j=1}^{l}(-1)^{k+1-i_{j}+l-j} \underbrace{e_{k+1-i_{j}}{ }^{k}}_{\mid} \otimes\left(w_{i_{i}} \wedge \cdots \wedge \hat{w}_{i_{j}} \wedge \cdots \wedge w_{i_{l}}\right) \wedge w_{k+1} \text {. }
\end{aligned}
$$

In what follows to simplify the notation, we will often omit the tensor sign between the graphical part and $\Lambda^{l} W_{k}$. Also, we will sometimes use a bullet labeled with a symmetric function instead of a box. 
Proposition 4.1 $r \mathbf{1}_{n}$ as defined above is a bicomplex, i.e.,

$$
d^{H} \circ d^{H}=0 \quad d^{V} \circ d^{H}+d^{H} \circ d^{V}=0 \quad d^{V} \circ d^{V}=0 .
$$

Before giving the proof, let us show how the beginning of this bicomplex looks like (see Fig. 1).

Proof The first formula $\left(d^{H}\right)^{2}=0$ is immediate from the definition, since $c \wedge c=0$.

For the next equation we have to check that the following square anticommutes:

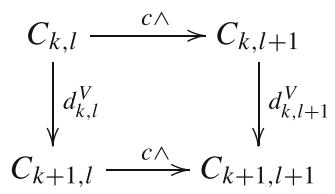

After moving all $c_{j}$ down, by using their centrality, the anticommutativity reduces to

$$
\sum_{i_{0}=1}^{k+1} w_{i_{0}} \wedge d_{k, l}^{V} c_{i_{0}}+\sum_{i_{0}=1}^{k} d_{k, l+1}^{V} \wedge w_{i_{0}} c_{i_{0}}=0
$$

or to the following identity in $\operatorname{Dot}\left(\mathcal{F}^{(k)} \mathcal{E}^{(k)} \mathbf{1}_{n}\right) \Lambda^{l} W_{k}$

$$
\begin{aligned}
& \sum_{i_{0}=1}^{k} c_{i_{0}} w_{i_{0}} \wedge w_{i_{1}} \wedge \cdots \wedge w_{i_{l}} \\
& -\sum_{j=0}^{l}(-1)^{k+1-i_{j}+l-j} \sum_{i_{0}=1}^{k} c_{i_{0}}\left(e_{k+1-i_{j}}\right)_{2} w_{i_{0}} \wedge \ldots \widehat{w}_{i_{j}} \cdots \wedge w_{i_{l}} \wedge w_{k+1} \\
= & \sum_{i_{0}=1}^{k+1} c_{i_{0}} w_{i_{0}} \wedge w_{i_{1}} \wedge \cdots \wedge w_{i_{l}} \\
& -\sum_{j=1}^{l}(-1)^{k+1-i_{j}+l-j} \sum_{i_{0}=1}^{k} c_{i_{0}}\left(e_{k+1-i_{j}}\right)_{2} w_{i_{0}} \wedge \ldots \widehat{w}_{i_{j}} \cdots \wedge w_{i_{l}} \wedge w_{k+1},
\end{aligned}
$$

where the lower index 2 indicates the stand where this endomorphism acts. This is easily seen to hold after accomplishing the second summand for $j=0$ to zero by means of the formula

$$
\sum_{i_{0}=1}^{k+1}(-1)^{k+1-i_{0}} c_{i_{0}}\left(e_{k+1-i_{0}}\right)_{2}=0 \in \operatorname{Dot}\left(\mathcal{F}^{(k)} \mathcal{E}^{(k)} \mathbf{1}_{n}\right)
$$

or pictorially

$$
\sum_{i=1}^{k+1}(-1)^{k+1-i} \underbrace{e_{k+1-i}^{k}}_{c_{i}}=0 .
$$

The formula (4.2) follows from the fact that $e_{k+1} \in \operatorname{Dot}\left(\mathcal{E}^{(k)} \mathbf{1}_{n}\right)\left(\right.$ or $e_{k+1} \in \operatorname{Dot}\left(\mathcal{F}^{(k)} \mathbf{1}_{n}\right)$ ) is zero and

$$
\begin{aligned}
\sum_{j=0}^{d}(-1)^{d-j} c_{j}\left(e_{d-j}\right)_{2} & =\sum_{j=0}^{d}(-1)^{j} \sum_{l=0}^{d-j}(-1)^{d-j-l}\left(e_{j}\right)_{1}\left(e_{d-j-l}\right)_{2}\left(h_{l}\right)_{2} \\
& =\left(c_{d}\right)_{1} \in \operatorname{Dot}\left(\mathcal{F}^{(r)} \mathcal{E}^{(s)} \mathbf{1}_{n}\right)
\end{aligned}
$$


for any $d, r$, and $s$, since $\sum_{i=0}^{p}(-1)^{i} e_{i} h_{p-i}=0$ for any $p>0$. Here $\left(c_{d}\right)_{1}=\left(c_{d}\right)_{\mathcal{F}^{(r)}}$ is equal to $(-1)^{d} e_{d}$ sitting on the first strand.

The proof of $d_{k, l}^{V} \circ d_{k-1, l}^{V}=0$ is based on the following identities
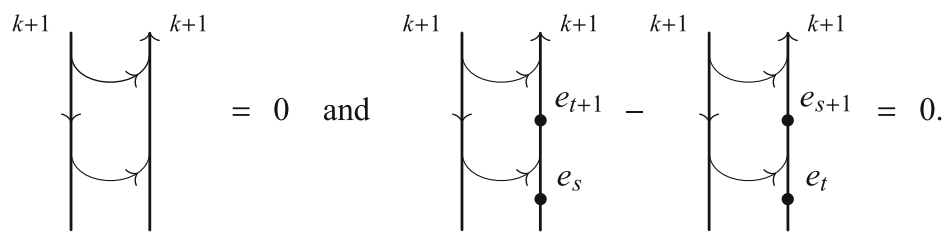

Let us explain this in more details. The formula (4.1) contains one summand without dots, let us call it $A$, and the other summands called $B$. Putting $A$ on top of $d_{k-1, l}^{V}$ is zero by the first identity printed above. Now any square resulting from putting $B$ on top of $d_{k-1, l}^{V}$ gives rise to the second identity with $t=k-i_{j}$ and $s=k-i_{j^{\prime}}$ or $s=0$.

The first identity can be proved as follows. By using the associativity of the trivalent vertices in the thick calculus ([11, Proposition 2.4]), we can attach the second horizontal line to the first one

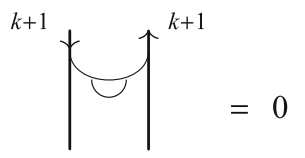

and then apply [11, Eq. (2.70)]. The proof of the second identity is similar after sliding $e_{t+1}$ and $e_{s+1}$ down by using the comultiplication rule in the ring of symmetric polynomials (or [11, Eq. (2.67)]).

\subsection{The Inverse Bicomplex}

The bicomplex $r^{-1} \mathbf{1}_{n}$ is defined as a kind of "left adjoint" of $r \mathbf{1}_{n}$. It has the form

$$
\begin{aligned}
& C_{00}^{L} \\
& \uparrow\left(d_{00}^{V}\right)^{L} \\
& C_{10}^{L} \stackrel{\left(d_{10}^{H}\right)^{L}}{\longleftarrow} C_{11}^{L} \\
& \uparrow\left(d_{10}^{V}\right)^{L} \uparrow\left(d_{11}^{V}\right)^{L} \\
& C_{20}^{L} \stackrel{\left(d_{20}^{H}\right)^{L}}{\longleftarrow} C_{21}^{L} \stackrel{\left(d_{21}^{H}\right)^{L}}{\longleftarrow} C_{22}^{L} \\
& \uparrow\left(d_{20}^{V}\right)^{L} \quad \uparrow\left(d_{21}^{V}\right)^{L} \quad \uparrow\left(d_{22}^{V}\right)^{L} \\
& C_{30}^{L} \stackrel{\left(d_{30}^{H}\right)^{L}}{\longleftarrow} C_{31}^{L} \stackrel{\left(d_{31}^{H}\right)^{L}}{\longleftarrow} C_{32}^{L} \stackrel{\left(d_{32}^{H}\right)^{L}}{\longleftarrow} C_{33}^{L} \\
& \uparrow\left(\begin{array}{llll}
\left.d_{30}^{V}\right)^{L} & \uparrow\left(d_{31}^{V}\right)^{L} & \uparrow\left(d_{32}^{V}\right)^{L} & \uparrow\left(d_{33}^{V}\right)^{L}
\end{array}\right.
\end{aligned}
$$

where

$$
C_{k, l}^{L}=\mathcal{F}^{(k)} \mathcal{E}^{(k)} \mathbf{1}_{n}\langle k n+k\rangle \otimes \Lambda^{l} \bar{W}_{k}=C_{k, l}^{R}
$$

with the total $q$-degree shift $\left\langle\frac{n^{2}}{2}+n\right\rangle$ and the homological shift $[-n / 2,-n / 2]$. Here $\bar{W}_{k}=$ $\operatorname{Span}_{\mathbb{Z}}\left\{\bar{w}_{1}, \ldots, \bar{w}_{k}\right\}$ and $\operatorname{deg}\left(\bar{w}_{j}\right)=2 j$. 
The differentials are obtained by rotating the original differentials by $\pi$. We have

$$
\begin{aligned}
\left(d_{k, l}^{H}\right)^{L}: C_{k, l+1}^{L} & \rightarrow C_{k, l}^{L} \\
\bar{w}_{i_{1}} \wedge \cdots \wedge \bar{w}_{i_{l+1}} & \mapsto \sum_{j=1}^{l}(-1)^{j-1} c_{j}^{L} \otimes \bar{w}_{i_{1}} \wedge \widehat{\bar{w}}_{i_{j}} \wedge \bar{w}_{i_{l+1}},
\end{aligned}
$$

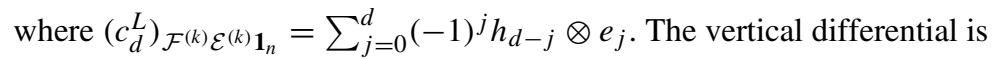

$$
\left(d_{k, l}^{V}\right)^{L}:=\left.(-1)^{l}\right|_{k+1} ^{k}: C_{k+1, l}^{L} \rightarrow C_{k, l}^{L}
$$

with $\alpha_{k, l}$ defined as before by (4.1).

\subsection{Intermediate Bicomplex}

Recall that $r \mathcal{E} \mathbf{1}_{n}$ is the bicomplex obtained by composing $r \mathbf{1}_{n+2}$ to the left of $\mathcal{E} \mathbf{1}_{n}$. Its "chain groups" are $\mathcal{F}^{(k)} \mathcal{E}^{(k)} \mathcal{E} \mathbf{1}_{n}\langle-k n-3 k\rangle \Lambda^{l} W_{k}$ with the total $q$-degree shift $\left\langle-\frac{n^{2}}{2}-3 n-4\right\rangle$ and homological shift $[n / 2+1, n / 2+1]$. We will denote them by $C_{k, l} \mathcal{E}$. The differentials are those of $r \mathbf{1}_{n+2}$ extended by identity on $\mathcal{E} \mathbf{1}_{n}$. The Euler characteristic of $r \mathcal{E} \mathbf{1}_{n}$ is

$$
\mathrm{r} E 1_{n}=q^{-\frac{n^{2}}{2}-3 n-4} \sum_{k=0}^{\infty}(-1)^{k} q^{-k n-2 k}\left(q^{-4} ; q^{-2}\right)_{k} F^{(k)} E^{(k+1)} 1_{n} .
$$

The intermediate bicomplex

$$
\left(r \mathcal{E} \mathbf{1}_{n}\right)^{\prime}=\left(\oplus_{k, l} C_{k, l}^{\prime}, d_{k, l}^{\prime H}, d_{k, l}^{\prime V}\right)
$$

is an indecomposable summand of $r \mathcal{E} \mathbf{1}_{n}$ defined as follows. The "chain groups" are

$$
C_{k, l}^{\prime}=\mathcal{F}^{(k)} \mathcal{E}^{(k+1)} \mathbf{1}_{n}\langle-k n-2 k\rangle \otimes \Lambda^{l} W_{k}^{\prime}
$$

with $W_{k}^{\prime}:=\operatorname{Span}_{\mathbb{Z}}\left\{w_{2}, \ldots, w_{k+1}\right\}$ and the same total shifts as for $r \mathcal{E} \mathbf{1}_{n}$.

The horizontal differential $d_{k, l}^{\prime H}$ sends $x$ to $c^{\prime} \wedge x$ where

$$
\begin{aligned}
c^{\prime} & :=\sum_{j=2}^{k+1} c_{j-1,1} w_{j} \\
& :=\sum_{j=2}^{k+1}\left(\sum_{\lambda, \mu \subset(j-1,1)}(-1)^{|\lambda|} N_{\lambda \mu}^{(j-1,1)} w_{j} \in \operatorname{Dot}\left(\mathcal{F}^{(k)} \mathcal{E}^{(k+1)} \mathbf{1}_{n}\right) W_{k}^{\prime} .\right.
\end{aligned}
$$

The vertical differential $d_{k, l}^{\prime V}: C_{k, l}^{\prime} \rightarrow C_{k+1, l}^{\prime}$ is defined as follows

$$
d_{k, l}^{\prime V}:=(-1)^{l} \overbrace{k+1}^{\alpha_{k, l}^{\prime}},
$$


where

$$
\alpha_{k, l}^{\prime}: \Lambda^{l} W_{k}^{\prime} \rightarrow \operatorname{Dot}\left(\mathcal{E}^{(k+1)} \mathbf{1}_{n}\right) \Lambda^{l} W_{k+1}^{\prime}
$$

is defined similar to (4.1). For $1<i_{1}<i_{2}<\cdots<i_{l} \leq k+1$, we have

$$
\begin{aligned}
& \alpha_{k, l}^{\prime}\left(w_{i_{1}} \wedge w_{i_{2}} \wedge \cdots \wedge w_{i_{l}}\right) \\
= & w_{i_{1}} \wedge \cdots \wedge w_{i_{l}}-\sum_{j=1}^{l}(-1)^{k-i_{j}+l-j} \underbrace{e_{k+2-i_{j}}} w_{i_{i}} \wedge \ldots \widehat{w}_{i_{j}} \cdots \wedge w_{i_{l}} \wedge w_{k+2} .
\end{aligned}
$$

It can be verified similarly to the previous case that $\left(r \mathcal{E} \mathbf{1}_{n}\right)^{\prime}$ is a bicomplex. The identity which replaces (4.2) here is

$$
\sum_{j=0}^{r}(-1)^{j} c_{r+1-j, 1}\left(e_{j}\right)_{2}=0 \in \operatorname{Dot}\left(\mathcal{F}^{(r)} \mathcal{E}^{(r+1)} \mathbf{1}_{n}\right) .
$$

It can be proven by using the comultiplication of Schur functions implying that

$$
\Delta\left(c_{k, 1}\right)=\sum_{i=0}^{k-1} c_{i} \otimes c_{k-i, 1}+\sum_{i=1}^{k} c_{i} \otimes c_{k+1-i}+\sum_{i=2}^{k+1} c_{i-1,1} \otimes c_{k+1-i} .
$$

\section{Chain Maps Between $r \mathcal{E} 1_{n}$ and $\left(r \mathcal{E} 1_{n}\right)^{\prime}$}

In this section, we will define the chain maps between $r \mathcal{E} \mathbf{1}_{n}$ and the intermediate complexes.

The map $f=\oplus_{k, l} f_{k, l}: r \mathcal{E} \mathbf{1}_{n} \rightarrow\left(r \mathcal{E} \mathbf{1}_{n}\right)^{\prime}$ is defined by

$$
f_{k, l}:=\underbrace{k+1}_{\substack{\beta_{k, l} \\ \mid>1}} C_{k, l} \mathcal{E} \rightarrow C_{k, l}^{\prime} \text {, }
$$

where $\beta_{k, l}: \Lambda^{l} W_{k} \rightarrow \operatorname{Dot}\left(\mathcal{F}^{(k)} \mathcal{E}^{(k)} \mathcal{E} \mathbf{1}_{n}\right) \Lambda^{l} W_{k}^{\prime}$ will be specified below. Recall that $W_{k}^{\prime}=$ $\operatorname{Span}_{\mathbb{Z}}\left\{w_{2}, \ldots, w_{k+1}\right\}$.

Let us first define a linear map

$$
\beta_{k}: W_{k} \rightarrow \operatorname{Dot}\left(\mathcal{F}^{(k)} \mathcal{E}^{(k)} \mathcal{E} \mathbf{1}_{n}\right) W_{k}^{\prime}
$$

by

$$
\left.\beta_{k}\left(w_{i}\right)=-w_{i}+\delta_{i, 1} \sum_{j=2}^{k+1} c_{j-1} w_{j}+(-1)^{k+1-i}\right\rfloor^{k} \uparrow^{e_{k+1-i}} \uparrow w_{k+1},
$$

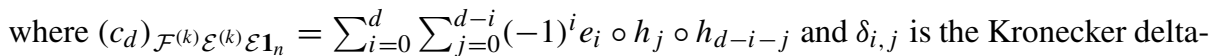
function.

In the matrix form, this map can be represented as follows

$$
M\left(\beta_{k}\right)=\left(\begin{array}{ccccc}
c_{1} & -\mathbf{1} & \ldots & 0 & 0 \\
c_{2} & 0 & \ldots & 0 & 0 \\
\ldots & \ldots & \ldots & \ldots & \ldots \\
c_{k-1} & 0 & \ldots & 0 & -\mathbf{1} \\
c_{k}+(-1)^{k}\left(e_{k}\right)_{2} & (-1)^{k-1}\left(e_{k-1}\right)_{2} & \ldots & \left(e_{2}\right)_{2} & -\left(e_{1}\right)_{2}
\end{array}\right),
$$


where as before $\left(e_{i}\right)_{2}$ means that $e_{i}$ sits on the second strand.

The map $\beta_{k}$ extends to an algebra homomorphism

$$
\beta_{k}: \Lambda^{\bullet} W_{k} \rightarrow \operatorname{Dot}\left(\mathcal{F}^{(k)} \mathcal{E}^{(k)} \mathcal{E} \mathbf{1}_{n}\right) \Lambda^{\bullet} W_{k}^{\prime}
$$

which in the matrix form can be written as $\beta_{k}=\oplus_{l=0}^{k} \beta_{k, l}$. This defines the matrix $\beta_{k, l}$ as the matrix of $(l, l)$-minors of the matrix $\beta_{k, 1}=M\left(\beta_{k}\right)$ or, alternatively

$$
\beta_{k, l}\left(w_{i_{1}} \wedge w_{i_{2}} \wedge \cdots \wedge w_{i_{l}}\right)=\beta_{k}\left(w_{i_{1}}\right) \wedge \beta_{k}\left(w_{i_{2}}\right) \wedge \cdots \wedge \beta_{k}\left(w_{i_{l}}\right) .
$$

We set $\beta_{k, 0}=1$.

For example, with the same notation as before

$$
\beta_{3,2}=\left(\begin{array}{ccc}
c_{2} & -c_{1} & \mathbf{1} \\
c_{1}\left(e_{2}\right)_{2}+c_{3}-\left(e_{3}\right)_{2} & -c_{1}\left(e_{1}\right)_{2} & \left(e_{1}\right)_{2} \\
c_{2}\left(e_{2}\right)_{2} & -c_{2}\left(e_{1}\right)_{2}+c_{3}-\left(e_{3}\right)_{2} & \left(e_{2}\right)_{2}
\end{array}\right),
$$

where each entry of this matrix is a determinant of the corresponding $2 \times 2$ matrix of $\beta_{3,1}$.

One can easily prove that

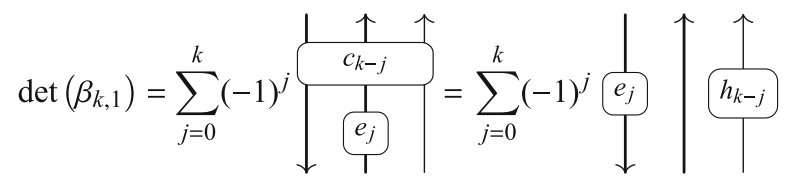

The inverse map $\bar{f}=\oplus_{k, l} \bar{f}_{k, l}:\left(r \mathcal{E} \mathbf{1}_{n}\right)^{\prime} \rightarrow r \mathcal{E} \mathbf{1}_{n}$ is defined by

$$
\bar{f}_{k, l}=(-1)^{k} \overbrace{\downarrow_{k}{ }_{\bar{\beta}_{k, l}}^{1}}^{1},
$$

where

$$
\bar{\beta}_{k, l}:=\operatorname{Adj}\left(\beta_{k, l}\right)=\operatorname{det}\left(\beta_{k, 1}\right) \beta_{k, l}^{-1}
$$

is given by the adjugate matrix, or the transpose of the cofactor matrix for $\beta_{k, l}$.

For example,

$$
\beta_{3,1}=\left(\begin{array}{ccc}
c_{1} & -\mathbf{1} & 0 \\
c_{2} & 0 & -\mathbf{1} \\
c_{3}-\left(e_{3}\right)_{2} & \left(e_{2}\right)_{2} & -\left(e_{1}\right)_{2}
\end{array}\right)
$$

and

$$
\bar{\beta}_{3,1}=\operatorname{Adj}\left(\beta_{3,1}\right)=\left(\begin{array}{ccc}
\left(e_{2}\right)_{2} & -\left(e_{1}\right)_{2} & \mathbf{1} \\
c_{2}\left(e_{1}\right)_{2}-c_{3}+\left(e_{3}\right)_{2} & -c_{1}\left(e_{1}\right)_{2} & c_{1} \\
c_{2}\left(e_{2}\right)_{2} & -c_{1}\left(e_{2}\right)_{2}-c_{3}+\left(e_{3}\right)_{2} & c_{2}
\end{array}\right)
$$

Proposition 5.1 The map $f \bar{f}:\left(r \mathcal{E} \mathbf{1}_{n}\right)^{\prime} \rightarrow\left(r \mathcal{E} \mathbf{1}_{n}\right)^{\prime}$ is equal to identity.

Proof We have

$$
f_{k, l} \bar{f}_{k, l}=(-1)^{k} \underbrace{\operatorname{det} \beta_{k, 1}}_{k \prod_{k+1}}=\left.\sum_{j=0}^{k}(-1)^{k+j}\right|_{k+1} ^{e_{k+1}}
$$


Proposition 5.2 The map $f: r \mathcal{E} \mathbf{1}_{n} \rightarrow\left(r \mathcal{E} \mathbf{1}_{n}\right)^{\prime}$ is a chain map between bicomplexes.

Proof Let us first check the commutativity of the horizontal square

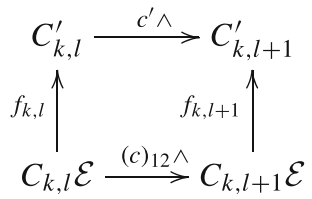

We have to show that for any $1 \leq i_{1}<i_{2}<\cdots<i_{l} \leq k$

$$
\begin{aligned}
& \sum_{i=2}^{k+1} c_{i-1,1} w_{i} \wedge \beta_{k}\left(w_{i_{1}}\right) \wedge \cdots \wedge \beta_{k}\left(w_{i_{l}}\right) \\
= & \sum_{i=1, i \neq i_{j}}^{k}\left(c_{i}\right)_{12} \beta_{k}\left(w_{i}\right) \wedge \beta_{k}\left(w_{i_{1}}\right) \wedge \cdots \wedge \beta_{k}\left(w_{i_{l}}\right) \in \operatorname{Dot}\left(\mathcal{F}^{(k)} \mathcal{E}^{(k)} \mathcal{E}\right) \Lambda^{l} W_{k} .
\end{aligned}
$$

Let us first assume $i_{1}>1$. After the substitution of maps, this identity can be rewritten as follows

$$
\begin{aligned}
& (-1)^{l} \sum_{i=2}^{k+1} c_{i-1,1} w_{i} \wedge w_{i_{1}} \wedge \cdots \wedge w_{i_{l}} \\
& +(-1)^{l-1} \sum_{j=1}^{l} \sum_{i=2}^{k+1}(-1)^{k+1-i_{j}}\left(e_{k+1-i_{j}}\right)_{2} c_{i-1,1} w_{i} \wedge \ldots \widehat{w}_{i_{j}} \cdots \wedge w_{i_{l}} \wedge w_{k+1} \\
& =(-1)^{l+1} \sum_{i=1}^{k}\left(c_{i}\right)_{12} w_{i} \wedge w_{i_{1}} \wedge \cdots \wedge w_{i_{l}}+(-1)^{l} \sum_{i=2}^{k+1}\left(c_{1}\right)_{12} c_{i-1} w_{i} \wedge w_{i_{1}} \wedge \cdots \wedge w_{i_{l}} \\
& +(-1)^{l-1} \sum_{j=1}^{l} \sum_{i=2}^{k+1}(-1)^{k+1-i_{j}}\left(c_{1}\right)_{12} c_{i-1}\left(e_{k+1-i_{j}}\right)_{2} w_{i} \wedge w_{i_{1}} \wedge \ldots \widehat{w}_{i_{j}} \cdots \wedge w_{i_{l}} \\
& +(-1)^{l} \sum_{j=0}^{l} \sum_{i_{0}=1, i_{0} \neq i_{j}}^{k}(-1)^{k+1-i_{j}}\left(c_{i_{0}}\right)_{12}\left(e_{k+1-i_{j}}\right)_{2} w_{i_{0}} \wedge \ldots \widehat{w}_{i_{j}} \cdots \wedge w_{i_{l}} \wedge w_{k+1} .
\end{aligned}
$$

After accomplishing the $j=0$ summand of the last term to zero by using (4.3) and applying twice

$$
c_{i-1,1}+\left(c_{i}\right)_{12}-\left(c_{1}\right)_{12} c_{i-1}=0
$$

it is not difficult to verify that it holds. The case $i_{1}=1$ reduces to the same identities and is left to the reader.

To see that $f_{k+1, l}\left(d_{k, l}^{V} \mathcal{E}\right)=d_{k, l}^{\prime V} f_{k, l}$, we need to verify that

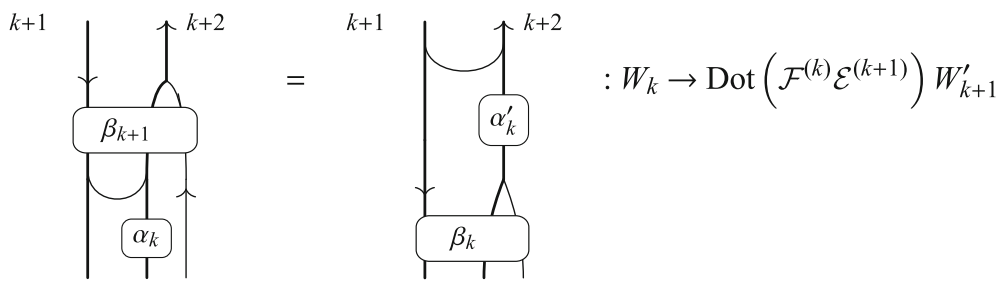


which can be easily seen after the substitution of maps. Hence, also the induced maps $\Lambda^{l} W_{k} \rightarrow \operatorname{Dot}\left(\mathcal{F}^{(k)} \mathcal{E}^{(k+1)}\right) \Lambda^{l} W_{k+1}^{\prime}$ have to coincide.

Theorem 5.3 The bicomplex $\left(r \mathcal{E} \mathbf{1}_{n}\right)^{\prime}$ is a strong deformation retract of $r \mathcal{E} \mathbf{1}_{n}$ in $\operatorname{Kom}(\dot{\mathcal{U}})$.

\section{Proof of Theorem 5.3}

This section is devoted to the proof of Theorem 5.3. After defining the homotopies, Propositions 6.1, 5.1 and 5.2 establish all properties of the strong deformation retract.

\subsection{Homotopies}

The horizontal homotopy $h_{k, l}^{H}: C_{k, l} \mathcal{E} \rightarrow C_{k, l-1} \mathcal{E}$ is defined as follows

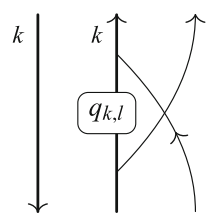

where $q_{k, l}: \Lambda^{l} W_{k} \rightarrow \operatorname{Dot}\left(\mathcal{E}^{(k-1)} \mathbf{1}_{n}\right) \Lambda^{l-1} W_{k}$

$$
q_{k, l}\left(w_{i_{1}} \wedge \cdots \wedge w_{i_{l}}\right)=\sum_{j=1}^{l}(-1)^{j-1} q_{k, 1}\left(w_{i_{j}}\right) w_{i_{1}} \wedge \ldots \widehat{w}_{i_{j}} \cdots \wedge w_{i_{l}}
$$

with $q_{k, 1}\left(w_{i}\right)=(-1)^{i-1} e_{k-i}$. The vertical homotopy $h_{k, l}^{V}: C_{k, l} \mathcal{E}$ to $C_{k-1, l} \mathcal{E}$ is set to be zero.

Now Theorem 5.3 reduces to the following proposition.

\section{Proposition 6.1 We have}

(1) $f_{k, l-1} h_{k, l}^{H}=0$;

(2) $h_{k, l-1}^{H} h_{k, l}^{H}=0$;

(3) $h_{k+1, l}^{H}\left(d_{k, l}^{V} \mathcal{E}\right)+\left(d_{k, l-1}^{V} \mathcal{E}\right) h_{k, l}^{H}=0$;

(4) $h_{k, l+1}^{H}\left(d_{k, l}^{H} \mathcal{E}\right)+\left(d_{k, l-1}^{H} \mathcal{E}\right) h_{k, l}^{H}=\mathbf{1}-\bar{f}_{k, l} f_{k, l}$.

Proof (1) The proof of the first identity requires to show that for any $1 \leq i, j \leq k$, we have

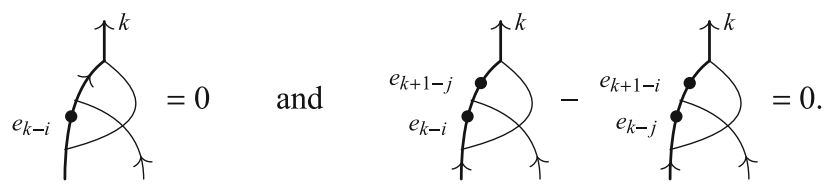

To prove the first statement, we use the associativity of the trivalent vertices.

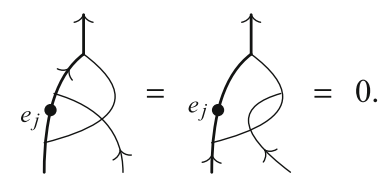


The second statement works similarly. We first move dots down, then cancel terms that coincide and finally use the same trick.

(2) Here we need to show that for all $0 \leq s, u \leq k-1$

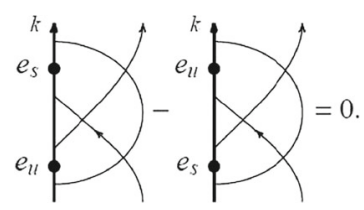

After sliding the upper $e_{i}$ 's down and using the invariance under the third Reidemeister move, the left hand side of this identity become

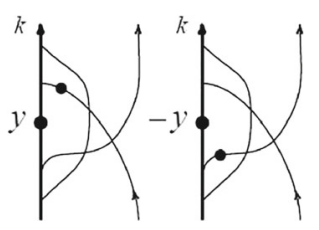

with $y:=e_{s-1} e_{u}-e_{u-1} e_{s}$. Using associativity as in case (1), we can easily see that it is zero.

(3) The third equation follows from the following identity:

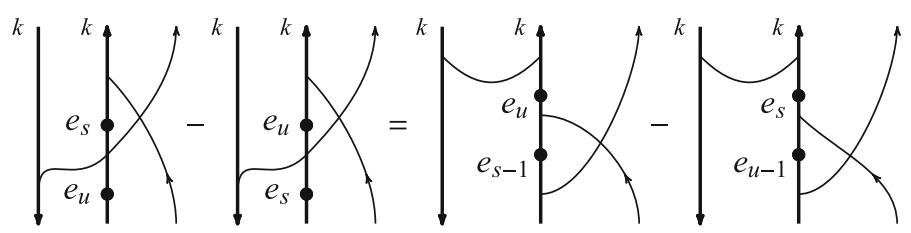

which holds for all $0 \leq s, u \leq k$ (here $e_{-1}$ are assumed to be zero). This identity is easy to prove by using the sliding rules from Appendix.

(4) Let us compute all terms of this equation. The " $d h$ "-part contains the diagonal term of the form

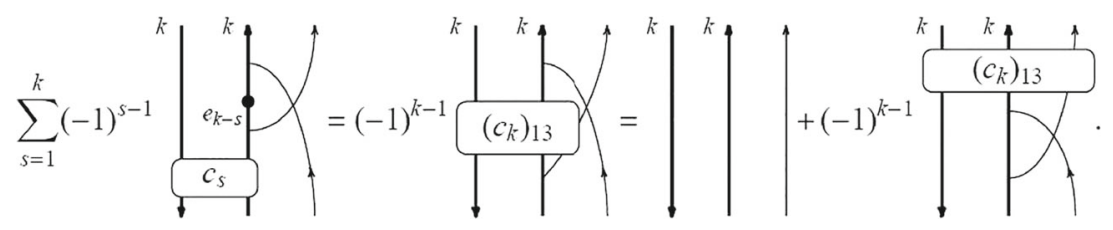


The off-diagonal terms of " $d h$ " and " $h d$ " are

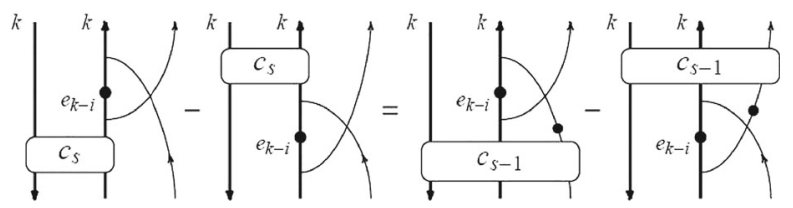

Finally, from the chain maps, we get

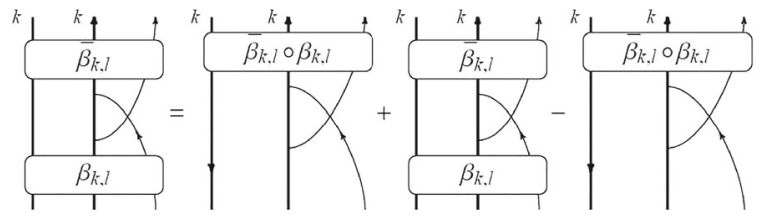

Due to (5.1), the first term in (6.2) cancels with the last term in (6.1) and the remaining terms cancel with the off-diagonal contributions from " $d h$ " and " $h d$."

Further details are left to the reader.

\section{Chain Maps Between $\mathcal{E} r 1_{n}$ and $\left(r \mathcal{E} 1_{n}\right)^{\prime}$}

Let us denote by $\mathcal{E} C_{k, l}$ the "chain groups" of the complex $\mathcal{E} r \mathbf{1}_{n}$, obtained by composing $\mathcal{E} \mathbf{1}_{n}$ with $r \mathbf{1}_{n}$. These groups are $\mathcal{E} \mathcal{F}^{(k)} \mathcal{E}^{(k)} \mathbf{1}_{n} \otimes \Lambda^{l} W_{k}\langle-k n-k\rangle$ with the total degree shift $-\frac{n^{2}}{2}-3 n-4$. The differentials are those of $r \mathbf{1}_{n}$ extended by identity on $\mathcal{E} \mathbf{1}_{n}$. The Euler characteristic of this complex is given by (4.4).

Define an algebra homomorphism

$$
\gamma_{k}: W_{k} \rightarrow \operatorname{Dot}\left(\mathcal{E F}^{(k-1)} \mathcal{E}^{(k-1)} \mathbf{1}_{n}\right) \otimes W_{k-1}^{\prime}
$$

by $\gamma_{k}\left(w_{1}\right)=-\sum_{j=2}^{k} c_{j-1} w_{j}$ and $\gamma_{k}\left(w_{i}\right)=w_{i}$ for $2 \leq i \leq k$. This extends to

$$
\gamma_{k}: \Lambda^{l} W_{k} \rightarrow \operatorname{Dot}\left(\mathcal{E} \mathcal{F}^{(k-1)} \mathcal{E}^{(k-1)} \mathbf{1}_{n}\right) \otimes \Lambda^{l} W_{k-1}^{\prime}
$$

defined by $\gamma_{k}\left(w_{i_{1}} \wedge \cdots \wedge w_{i_{l}}\right)=\gamma_{k}\left(w_{i_{1}}\right) \wedge \cdots \wedge \gamma_{k}\left(w_{i_{l}}\right)$. We will also need another map $a_{k}: W_{k} \rightarrow \operatorname{Dot}\left(\mathcal{E} \mathcal{F}^{(k-1)} \mathcal{E}^{(k-1)} \mathbf{1}_{n}\right)$ with

$$
\left.a_{k}\left(w_{i}\right)=(-1)^{k-i}\left(e_{k-i}\right)_{3}=(-1)^{k-i} \uparrow\right\rfloor_{\mid}^{k-1} \uparrow^{k-1}
$$

Let $\gamma_{k, l}: \Lambda^{\bullet} W_{k} \rightarrow \operatorname{Dot}\left(\mathcal{E} \mathcal{F}^{(k-1)} \mathcal{E}^{(k-1)} \mathbf{1}_{n}\right) \otimes \Lambda^{\bullet-1} W_{k}^{\prime}$ be the derivation along the homomorphism $\gamma_{k}$ induced by $a_{k}$, i.e., $\gamma_{k, 0}(1)=0$ and for $l \geq 0$ we have

$$
\gamma_{k, l}\left(w_{i_{1}} \wedge \cdots \wedge w_{i_{l}}\right)=\sum_{j=1}^{l}(-1)^{j-1} a_{k}\left(w_{i_{j}}\right) \otimes \gamma_{k}\left(w_{i_{1}} \wedge \ldots \widehat{w}_{i_{j}} \cdots \wedge w_{i_{l}}\right)
$$


Then we define the map

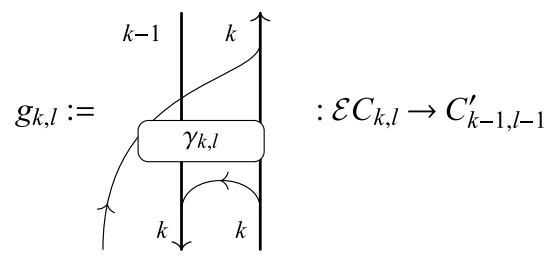

and set $g=\oplus_{k, l} g_{k, l}: \mathcal{E} r \mathbf{1}_{n} \rightarrow\left(r \mathcal{E} \mathbf{1}_{n}\right)^{\prime}$. The inverse map $p=\oplus_{k, l} p_{k, l}:\left(r \mathcal{E} \mathbf{1}_{n}\right)^{\prime} \rightarrow \mathcal{E} r \mathbf{1}_{n}$ is defined as follows

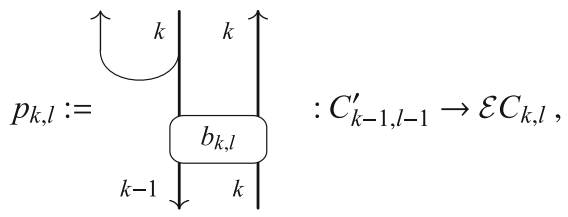

where $b_{k, l}\left(w_{i_{2}} \wedge \ldots w_{i_{l}}\right)=(-1)^{k-1} \sum_{i=1}^{k} c_{i-1} \otimes w_{i} \wedge w_{i_{2}} \wedge \cdots \wedge w_{i_{l}}$.

Theorem 7.1 The bicomplex $\left(r \mathcal{E} \mathbf{1}_{n}\right)^{\prime}$ is a strong deformation retract of the bicomplex $\mathcal{E} r \mathbf{1}_{n}$ in $\operatorname{Kom}(\dot{\mathcal{U}})$.

\section{Proof of Theorem 7.1}

We will split the proof of Theorem 7.1 into lemmas and prove them separately.

Lemma $8.1 g: \mathcal{E} r \mathbf{1}_{n} \rightarrow\left(r \mathcal{E} \mathbf{1}_{n}\right)^{\prime}$ is a chain map between bicomplexes, i.e.,

$$
\begin{aligned}
& d_{k-1, l-1}^{\prime H} g_{k, l}=g_{k, l+1}\left(\mathcal{E} d_{k, l}^{H}\right), \\
& d_{k-1, l-1}^{\prime V} g_{k, l}=f_{k+1, l}\left(\mathcal{E} d_{k, l}^{V}\right) .
\end{aligned}
$$

Proof Let us check the commutativity of a general horizontal square

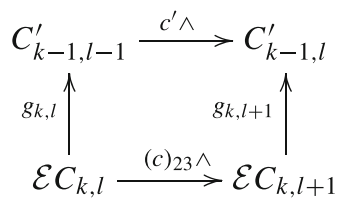

We assume $k \geq 2$, otherwise there is nothing to check. We start with

$$
\mathcal{E F}^{(k)} \mathcal{E}^{(k)} w_{i_{1}} \wedge \cdots \wedge w_{i_{l}} \in \mathcal{E} C_{k, l}
$$

and first apply the map $g_{k, l}$ followed by $d_{k-1, l-1}^{\prime H}$. Then we get

$$
\sum_{t=2}^{k} \sum_{j=1}^{l}(-1)^{j-1+k-i_{j}} c_{t-1,1}\left(e_{k-i_{j}}\right)_{3} w_{t} \wedge \gamma_{k}\left(w_{i_{1}} \wedge \ldots \widehat{w}_{i_{j}} \ldots \wedge w_{i_{l}}\right) \text {. }
$$


Applying first the differential and then the map, we get

$$
\sum_{j=0}^{l} \sum_{i_{0}=1}^{k}(-1)^{j+k-i_{j}}\left(c_{i_{0}}\right)_{23}\left(e_{k-i_{j}}\right)_{3} \gamma_{k}\left(w_{i_{0}} \wedge w_{i_{1}} \wedge \ldots \widehat{w}_{i_{j}} \ldots \wedge w_{i_{l}}\right) .
$$

We claim that these expressions are equal in $\operatorname{Dot}\left(\mathcal{F}^{(k-1)} \mathcal{E}^{(k)} \mathbf{1}_{n}\right) \Lambda^{l} W_{k-1}^{\prime}$. Indeed, assume $1 \neq i_{1}<i_{2}<\cdots<i_{l}$ and $t \neq i_{s}$ for all $1 \leq s \leq l$. Then collecting the coefficients in front of $w_{t} \wedge w_{i_{1}} \wedge \ldots \widehat{w}_{i_{j}} \cdots \wedge w_{i_{l}}$ with $j>1$ in the both formulas we get $(-1)^{j+k-i_{j}}\left(e_{k-i_{j}}\right)_{3}$ times

$$
c_{t-1,1}+\left(c_{t}\right)_{23}-\left(c_{1}\right)_{23} c_{t-1}=0,
$$

where the last term comes from setting $i_{0}=1$ and picking the $t$ th summand in $\gamma_{k}\left(w_{1}\right)=$ $-\sum_{j=2}^{k} c_{j-1} w_{j}$.

Allowing $i_{1}=1$, but $i_{j} \neq 1$, leads to the same identity.

Let us consider the case $i_{j}=1$. Collecting the coefficients of $w_{t} \wedge w_{i_{2}} \wedge \cdots \wedge w_{i_{l}}$ in (8.1), we get

$$
(-1)^{k-1} c_{t-1,1}\left(e_{k-1}\right)_{3}+\sum_{j=2}^{l}(-1)^{k-i_{j}+1}\left(e_{k-i_{j}}\right)_{3}\left(c_{t-1,1} c_{i_{j}-1}-c_{t-1} c_{i_{j}-1,1}\right)
$$

and in (8.2)

$$
\begin{aligned}
& (-1)^{k}\left(c_{t}\right)_{23}\left(e_{k-1}\right)_{3}-\sum_{j=2}^{l}(-1)^{k-i_{j}+1}\left(e_{k-i_{j}}\right)_{3}\left(c_{t}\right)_{23} c_{i_{j}-1} \\
& -\sum_{p \neq 1, i_{2}, \ldots, i_{l}}(-1)^{k-p}\left(e_{k-p}\right)_{3}\left(c_{p}\right)_{23} c_{t-1} .
\end{aligned}
$$

Using (8.3) few times, we can reduce the claim to (4.3).

Let us consider the following vertical square

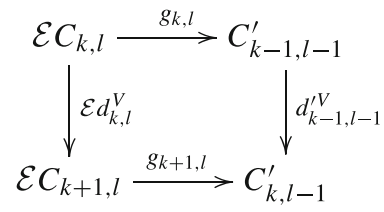

Similar considerations as before lead in all cases to the following true identity:

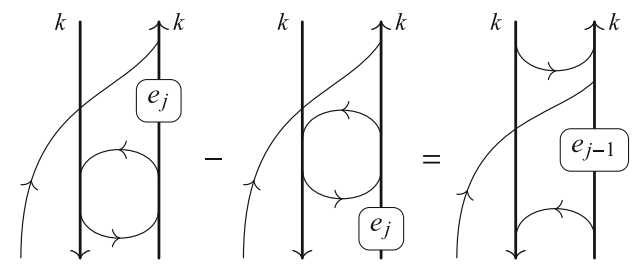

Lemma 8.2 We have $g p=1 \in \operatorname{End}\left(C_{k-1, l-1}^{\prime}\right)$. 
Proof Putting $g$ on the top of $p$ we get for any $1<i_{2}<\cdots<i_{l}$

$$
g p\left(w_{i_{2}} \wedge \cdots \wedge w_{i_{l}}\right)=\sum_{j=1}^{l} \sum_{i_{1}=1}^{k}(-1)^{j-i_{j}}
$$

which is equal to

$$
+\sum_{j=2}^{l} \sum_{i=2, i \neq i_{2}, \ldots, i_{l}}^{k}(-1)^{i_{j}-j-1} w_{i=1, i \neq i_{2}, \ldots, i_{l}}^{k} \wedge \wedge w_{i_{l}}
$$

After cancellation, we get

$$
g p\left(w_{i_{2}} \wedge \cdots \wedge w_{i_{l}}\right)=\sum_{i=1}^{k}(-1)^{i-1}
$$

Using the Reidemeister move listed in Appendix, we can see that the only non-zero term without bubbles is the desired identity, and all the bubble terms cancel since

$$
\sum_{i=0}^{k-1}(-1)^{i} c_{i}^{k-2}=0
$$

where the last identity is equivalent to (4.2). Here we are again using centrality of $c_{i}$ 's. 


\subsection{Horizontal Homotopy}

Let $q_{k, 1}: W_{k} \rightarrow \operatorname{Dot}\left(\mathcal{E}^{(k-1)} \mathbf{1}_{n}\right)$ be the map defined by $q_{k, 1}\left(w_{i}\right)=(-1)^{i-1} e_{k-i}$. Then let $q_{k, l}: \Lambda^{l} W_{k} \rightarrow \operatorname{Dot}\left(\mathcal{E}^{(k-1)} \mathbf{1}_{n}\right) \otimes \Lambda^{l-1} W_{k}$ be the derivation induced by $q_{k, 1}$, i.e.,

$$
q_{k, l}\left(w_{i_{1}} \wedge \cdots \wedge w_{i_{l}}\right)=\sum_{j=1}^{l}(-1)^{j-1} q_{k, 1}\left(w_{i_{j}}\right) w_{i_{1}} \wedge \ldots \widehat{w}_{i_{j}} \cdots \wedge w_{i_{l}} .
$$

We set

$$
\mathrm{h}_{k, l}^{H}:=>_{k \downarrow}^{k}
$$

Lemma 8.3 We have

$$
\begin{aligned}
g_{k, l-1} \mathrm{~h}_{k, l}^{H} & =0, \\
\mathrm{~h}_{k, l-1}^{H} \circ \mathrm{h}_{k, l}^{H} & =0, \\
\mathrm{~h}_{k+1, l}^{H}\left(\mathcal{E} d_{k, l}^{V}\right)+\left(\mathcal{E} d_{k, l-1}^{V}\right) \mathrm{h}_{k, l}^{H} & =0 .
\end{aligned}
$$

Proof Equation (8.5) reduces to
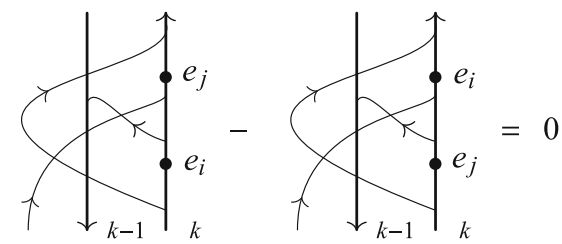

for $1 \leq i<j \leq k-1$. Indeed, moving the line starting at the lowest left corner up through the 3 -valent vertex (note that the bubble terms cancel) and then moving the $e_{j}$ and $e_{i}$ to the middle of the strand, we get that the left hand side is equal to

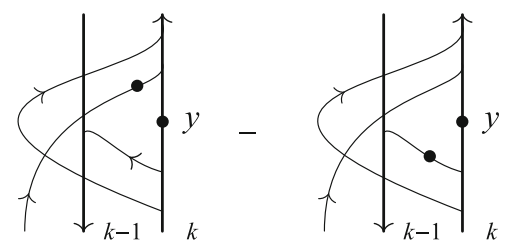

where $y:=e_{j-1} e_{i}-e_{i-1} e_{j}$. Now using the associativity and the invariance under the 3. Reidemeister move with all strands going in the same direction, we can see that both summands vanish. 
Equation (8.6) reduces to the following identity

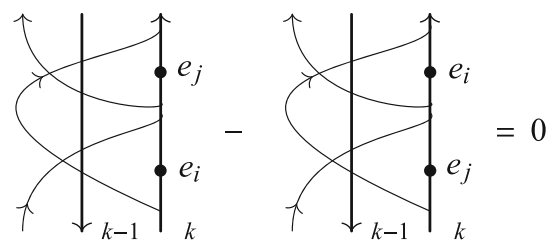

for $1 \leq i<j \leq k-1$, which can be proved similarly.

Finally, (8.7) follows from
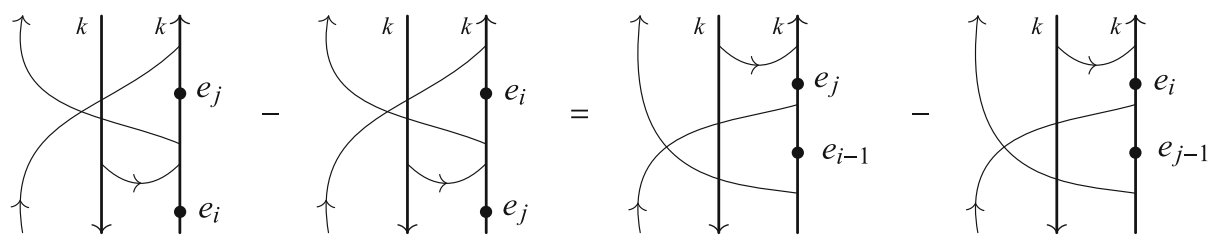

which holds for any $0 \leq i<j \leq k$.

\subsection{Vertical Homotopy}

We set

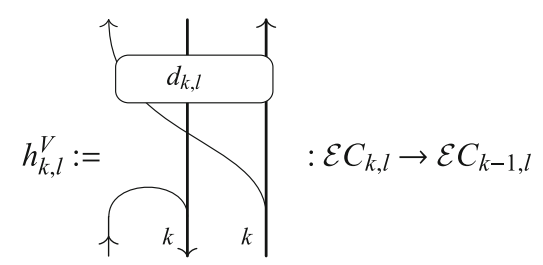

where $d_{k, l}: \Lambda^{l} W_{k} \rightarrow \operatorname{Dot}\left(\mathcal{E} \mathcal{F}^{(k)} \mathcal{E}^{(k)} \mathbf{1}_{n}\right) \otimes \Lambda^{l} W_{k-1}$ is given by

$$
\begin{aligned}
d_{k, 0}(1)= & (-1)^{k-1}\left(c_{k-1}\right)_{12}=(-1)^{k-1} \underbrace{\wedge}_{|+|} \uparrow \\
d_{k, 1}\left(w_{i}\right)= & (-1)^{k}\left(c_{k-1}\right)_{12} w_{i}+(-1)^{i-1}\left(e_{k-i}\right)_{3} \\
& \times\left(w_{1}+c_{1} w_{2}+\cdots+c_{k-2} w_{k-1}\right) \text { for } i<k \\
d_{k, 1}\left(w_{k}\right)= & (-1)^{k-1}\left(w_{1}+c_{1} w_{2}+\cdots+c_{k-2} w_{k-1}\right) \quad \text { and } \\
d_{k, l}\left(w_{i_{1}} \wedge \ldots w_{i_{l}}\right)= & (-1)^{l-1}\left((-1)^{k}\left(c_{k-1}\right)_{12}\right)^{-l+1} d_{k, 1}\left(w_{i_{1}}\right) \wedge \cdots \wedge d_{k, 1}\left(w_{i_{l}}\right) \\
= & (-1)^{l-1}\left((-1)^{k}\left(c_{k-1}\right)_{12} w_{i_{1}} \wedge \cdots \wedge w_{i_{l}}\right. \\
& \left.+\sum_{j=1}^{l}(-1)^{i_{j}-1}\left(e_{k-i_{j}}\right)_{3} w_{i_{1}} \wedge \ldots(X)_{j} \cdots \wedge w_{i_{l}}\right)
\end{aligned}
$$

where $X:=\sum_{i=1}^{k-1} c_{i-1} w_{i}$ and $(X)_{j}$ means that we replace $w_{i_{j}}$ with $X$. As before the lower indices indicate the strands on which the morphism is acting. 
Let us illustrate this definition with few examples.

$$
\begin{aligned}
& d_{3,1}=\left(\begin{array}{ccc}
-\left(c_{2}\right)_{12}+\left(e_{2}\right)_{3} & -\left(e_{1}\right)_{3} & 1 \\
\left(e_{2}\right)_{3} c_{1} & -\left(c_{2}\right)_{12}-\left(e_{1}\right)_{3} c_{1} & c_{1}
\end{array}\right) \\
& d_{3,1} \wedge d_{3,1}=\left(\operatorname{det}\left(\begin{array}{cc}
-\left(c_{2}\right)_{12}+\left(e_{2}\right)_{3} & -\left(e_{1}\right)_{3} \\
\left(e_{2}\right)_{3} c_{1} & -\left(c_{2}\right)_{12}-\left(e_{1}\right)_{3} c_{1}
\end{array}\right)\right. \text {, } \\
& \left.\operatorname{det}\left(\begin{array}{cc}
-\left(c_{2}\right)_{12}+\left(e_{2}\right)_{3} & \mathbf{1} \\
\left(e_{2}\right)_{3} c_{1} & c_{1}
\end{array}\right),\left(\begin{array}{cc}
-\left(e_{1}\right)_{3} & \mathbf{1} \\
-\left(c_{2}\right)_{12}-\left(e_{1}\right)_{3} c_{1} & c_{1}
\end{array}\right)\right) \text {. }
\end{aligned}
$$

Hence, we get

$$
\begin{aligned}
d_{3,1} \wedge d_{3,1} & =-\left(c_{2}\right)_{12}\left(-c_{2}, c_{1}, \mathbf{1}\right) \\
d_{3,2} & =\left(c_{2},-c_{1}, \mathbf{1}\right) .
\end{aligned}
$$

Similarly, $d_{k, k-1}=\left(c_{k-1},-c_{k-2}, \ldots,(-1)^{k-2} c_{1},(-1)^{k-1} \mathbf{1}\right)$.

\section{Lemma 8.4 We have}

$$
\begin{aligned}
g_{k-1, l} h_{k, l}^{V} & =0, \\
h_{k, l}^{V} p_{k, l} & =0, \\
h_{k-1, l}^{V} \circ h_{k, l}^{V} & =0, \\
\mathrm{~h}_{k-1, l}^{H} h_{k, l}^{V}+h_{k, l-1}^{V} \mathrm{~h}_{k, l}^{H} & =0, \\
h_{k, l+1}^{V}\left(\mathcal{E} d_{k, l}^{H}\right)+\left(\mathcal{E} d_{k-1, l}^{H}\right) h_{k, l}^{V} & =0, \\
\mathrm{~h}_{k, l+1}^{H}\left(\mathcal{E} d_{k, l}^{H}\right)+h_{k+1, l}^{V}\left(\mathcal{E} d_{k, l}^{V}\right)+\left(\mathcal{E} d_{k, l-1}^{H}\right) \mathrm{h}_{k, l}^{H}+\left(\mathcal{E} d_{k-1, l}^{V}\right) h_{k, l}^{V} & =\mathbf{1}-p_{k, l} g_{k, l} .
\end{aligned}
$$

Proof The proof of the last equality is based on the two identities given in Lemmas 5, 6 in Appendix. The rest is similar to the previous computations and hence left to the reader.

\subsection{Proof of Lemma 1.4}

Thus, we proved that the map

$$
\kappa_{\mathcal{E}}: \mathcal{E} r \mathbf{1}_{n} \stackrel{g}{\longrightarrow}\left(r \mathcal{E} \mathbf{1}_{n}\right)^{\prime} \stackrel{\bar{f}}{\longrightarrow} r \mathcal{E} \mathbf{1}_{n}
$$

has a homotopy inverse

$$
\bar{\kappa}_{\mathcal{E}}: \mathcal{E} r \mathbf{1}_{n} \longleftarrow{ }_{p}\left(r \mathcal{E} \mathbf{1}_{n}\right)^{\prime} \longleftarrow{ }_{f} r \mathcal{E} \mathbf{1}_{n} .
$$

To construct $\kappa_{\mathcal{F}}$, we apply the symmetry $\sigma \omega$ to $\kappa_{\mathcal{E}}$ assuming that Theorem 1.1 (Symmetry) holds. We get

$$
\bar{\kappa}_{\mathcal{F}}: r \mathcal{F} \mathbf{1}_{n+2} \stackrel{\sigma \omega(g)}{\longrightarrow}\left(r \mathcal{E} \mathbf{1}_{n}\right)^{\prime} \stackrel{\sigma \omega(\bar{f})}{\longrightarrow} \mathcal{F} r \mathbf{1}_{n+2}
$$

together with its homotopy inverse

$$
\kappa_{\mathcal{F}}: r \mathcal{F} \mathbf{1}_{n+2} \underset{\sigma \omega(p)}{\leftarrow}\left(r \mathcal{E} \mathbf{1}_{n}\right)^{\prime} \underset{\sigma \omega(f)}{\leftarrow} \mathcal{F} r \mathbf{1}_{n+2}
$$




\subsection{Proof of Theorem 1.1 (Centrality)}

We assume that Theorem 1.1 (Symmetry) holds.

Since any 1-morphism in $\dot{\mathcal{U}}$ is a direct sum of compositions of $\mathcal{E}\langle t\rangle$ and $\mathcal{F}\left\langle t^{\prime}\right\rangle$ with $t, t^{\prime} \in \mathbb{Z}$, it is enough to check the statement for the generators. Lemma 1.4 defines the maps $\kappa_{\mathcal{E}}$ and $\kappa_{\mathcal{F}}$ as well as their homotopy inverses. Applying symmetry, we can define $\eta_{\mathcal{F}}=\psi\left(\bar{\kappa}_{\mathcal{F}}\right)$ and $\eta_{\mathcal{E}}=\psi\left(\kappa_{\mathcal{E}}\right)$. The details are left to the reader.

\subsection{Comments on the Naturality of Maps $\kappa_{X}$}

To prove Conjecture 1.2 (Naturality), we need to show that for any chain map $f: X \rightarrow Y$, the squares below commute up to chain homotopy.
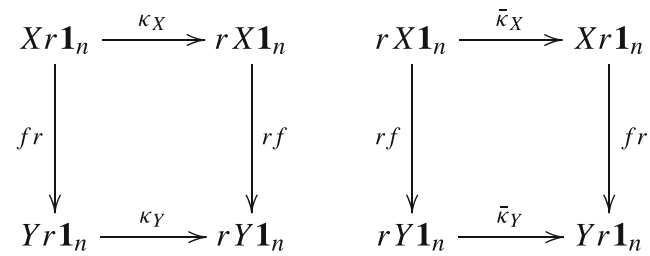

The commutativity of similar diagrams for $\eta_{X}$ will follow then by applying symmetry functors. It is enough to check the commutativity for short chain complexes $f: X \rightarrow Y$, where $X, Y$ are $\mathcal{E} \mathbf{1}_{n}, \mathcal{E}^{2} \mathbf{1}_{n}, \mathcal{F} \mathcal{E} \mathbf{1}_{n}$ or $\mathbf{1}_{n}$ and the differential is one of the generating 2-morphisms: dot, crossing, cup or cap. We leave this problem for future investigations.

\section{Symmetry 2-functors}

The 2-category $\mathcal{U}$ has the symmetry group $\mathcal{G}=(\mathbb{Z} / 2 \mathbb{Z})^{3}$ generated by the involutive 2 functors $\omega, \sigma, \psi$ described below.

\subsection{2-functor $\omega$}

Consider the operation on the diagrammatic calculus that rescales the crossing

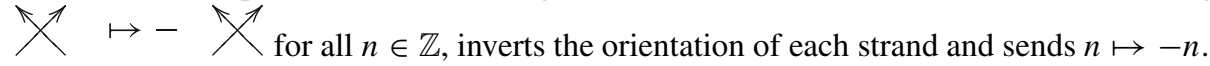

This gives a strict invertible 2-functor $\omega: \mathcal{U} \rightarrow \mathcal{U}$ given by

$$
\begin{aligned}
\omega: \mathcal{U} & \rightarrow \mathcal{U} \\
n & \mapsto-n \\
\mathbf{1}_{m} \mathcal{E}^{\alpha_{1}} \mathcal{F}^{\beta_{1}} \mathcal{E}^{\alpha_{2}} \ldots \mathcal{E}^{\alpha_{k}} \mathcal{F}^{\beta_{k}} \mathbf{1}_{n}\{s\} & \mapsto \mathbf{1}_{-m} \mathcal{F}^{\alpha_{1}} \mathcal{E}^{\beta_{1}} \mathcal{F}^{\alpha_{2}} \ldots \mathcal{F}^{\alpha_{k}} \mathcal{E}^{\beta_{k}} \mathbf{1}_{-n}\{s\} .
\end{aligned}
$$

This 2-functor extends to a 2-functor

$$
\begin{aligned}
\omega: \operatorname{Kom}(\mathcal{U}) & \rightarrow \operatorname{Kom}(\mathcal{U}) \\
n & \mapsto-n \\
\left(X^{\bullet}, d\right) & \mapsto \cdots \longrightarrow \omega\left(X^{i-1}\right) \stackrel{\omega\left(d_{i-1}\right)}{\longrightarrow} \omega\left(X^{i}\right) \stackrel{\omega\left(d_{i}\right)}{\longrightarrow} \omega\left(X^{i+1}\right) \longrightarrow \cdots \\
f_{i}: X^{\bullet} \rightarrow Y^{\bullet} & \mapsto \omega\left(f_{i}\right): \omega\left(X^{\bullet}\right) \rightarrow \omega\left(Y^{\bullet}\right) .
\end{aligned}
$$

Finally, this operation extends to the Karoubi envelope $\dot{\mathcal{U}}$. The images of the idempotents $e_{a}, e_{a}^{\prime}$ under the 2-functor $\omega$ are again idempotents. 


\subsection{2-functor $\sigma$}

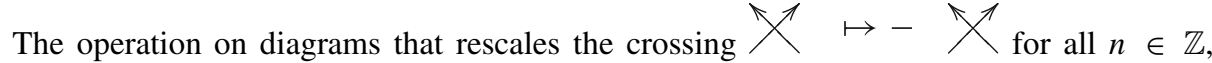
reflects a diagram across the vertical axis, and sends $n$ to $-n$ leaves invariant the relations on the 2-morphisms of $\mathcal{U}$.

This operation is contravariant for composition of 1-morphisms, covariant for composition of 2-morphisms, and preserves the degree of a diagram. This symmetry gives an invertible 2-functor

$$
\begin{aligned}
\sigma: \mathcal{U} & \rightarrow \mathcal{U}^{\mathrm{op}} \\
n & \mapsto-n \\
\mathbf{1}_{m} \mathcal{E}^{\alpha_{1}} \mathcal{F}^{\beta_{1}} \mathcal{E}^{\alpha_{2}} \ldots \mathcal{E}^{\alpha_{k}} \mathcal{F}^{\beta_{k}} \mathbf{1}_{n}\{s\} & \mapsto \mathbf{1}_{-n} \mathcal{F}^{\beta_{k}} \mathcal{E}^{\alpha_{k}} \mathcal{F}^{\beta_{k-1}} \ldots \mathcal{F}^{\beta_{1}} \mathcal{E}^{\alpha_{1}} \mathbf{1}_{-m}\{s\}
\end{aligned}
$$

that acts on 2-morphisms via the symmetry described above. This 2-functor extends to a 2-functor

$$
\begin{aligned}
\sigma: \operatorname{Kom}(\mathcal{U}) & \rightarrow \operatorname{Kom}(\mathcal{U}) \\
n & \mapsto-n \\
\left(X^{\bullet}, d\right) & \mapsto \cdots \longrightarrow \sigma\left(X^{i-1}\right) \stackrel{\sigma\left(d_{i-1}\right)}{\longrightarrow} \sigma\left(X^{i}\right) \stackrel{\sigma\left(d_{i}\right)}{\longrightarrow} \sigma\left(X^{i+1}\right) \longrightarrow \cdots \\
f_{i}: X^{\bullet} \rightarrow Y^{\bullet} & \mapsto \sigma\left(f_{i}\right): \sigma\left(X^{\bullet}\right) \rightarrow \sigma\left(Y^{\bullet}\right) .
\end{aligned}
$$

Note that $\sigma$ acts contravariantly on 1-morphisms in $\operatorname{Kom}(\mathcal{U})$.

Furthermore, $\sigma$ extends to a 2-functor $\dot{\mathcal{U}} \rightarrow \dot{\mathcal{U}}^{\text {op }}$. A formula for $\sigma\left(e_{a}\right)$ is given in [11, Eq. (5.59)].

\subsection{2 -functor $\psi$}

This operation reflects across the horizontal axis and invert orientation. This gives an invertible 2-functor defined by

$$
\begin{aligned}
\psi: \mathcal{U} & \rightarrow \mathcal{U}^{\text {co }} \\
n & \mapsto n \\
\mathbf{1}_{m} \mathcal{E}^{\alpha_{1}} \mathcal{F}^{\beta_{1}} \mathcal{E}^{\alpha_{2}} \ldots \mathcal{E}^{\alpha_{k}} \mathcal{F}^{\beta_{k}} \mathbf{1}_{n}\{s\} & \mapsto \mathbf{1}_{m} \mathcal{E}^{\alpha_{1}} \mathcal{F}^{\beta_{1}} \mathcal{E}^{\alpha_{2}} \ldots \mathcal{E}^{\alpha_{k}} \mathcal{F}^{\beta_{k}} \mathbf{1}_{n}\{-s\}
\end{aligned}
$$

and on 2-morphisms $\psi$ reflects the diagrams representing summands across the $x$-axis and inverts the orientation.

Since $\psi$ is contravariant on 2-morphisms in $\mathcal{U}$, this 2 -functor extends to a 2 -functor

$$
\begin{aligned}
\psi: \operatorname{Kom}(\mathcal{U}) & \rightarrow \operatorname{Kom}(\mathcal{U}) \\
n & \mapsto n \\
\left(X^{\bullet}, d\right) & \mapsto \cdots \longrightarrow \psi\left(X^{i+1}\right) \stackrel{\psi\left(d_{i}\right)}{\longrightarrow} \psi\left(X^{i}\right) \stackrel{\psi\left(d_{i-1}\right)}{\longrightarrow} \psi\left(X^{i-1}\right) \longrightarrow \\
f_{i}: X^{\bullet} \rightarrow Y^{\bullet} & \mapsto \psi\left(f_{i}\right): \psi\left(Y^{\bullet}\right) \rightarrow \psi\left(X^{\bullet}\right) .
\end{aligned}
$$

Notice that $\psi$ inverts the homological degree so that $\psi$ acts on a complex $\left(X^{\bullet} \mathbf{1}_{n}, \partial\right)$ in $\operatorname{Kom}(\mathcal{U})$ by $\psi\left(X^{i}\right)=\left(\psi X^{\bullet}\right)^{-i}$.

These 2-functors commute with each other 'on-the-nose'

$$
\omega \sigma=\sigma \omega, \quad \sigma \psi=\psi \sigma, \quad \omega \psi=\psi \omega .
$$

As before, $\psi$ extends to Karoubi envelopes providing a 2-functor $\dot{\mathcal{U}} \rightarrow \dot{\mathcal{U}}^{\text {co }}$. 


\section{Symmetries of the Ribbon Bicomplex}

In this section, we describe the behavior of the ribbon bicomplex under the symmetry 2functors.

\subsection{The Image Under $\omega$}

The "chain groups" of the bicomplex $\omega\left(r \mathbf{1}_{-n}\right)$ are $\omega\left(C_{k, l}\right):=\mathcal{E}^{(k)} \mathcal{F}^{(k)} \mathbf{1}_{n}\langle k n-k\rangle \otimes \Lambda^{l} W_{k}, \quad W_{k}=\operatorname{Span}_{\mathbb{Z}}\left\{w_{1}, \ldots, w_{k}\right\}, \quad \operatorname{deg}\left(w_{j}\right)=-2 j$ with the total shifts $\left\langle-\frac{n^{2}}{2}+n\right\rangle$ and $[n / 2, n / 2]$. The horizontal differential $\omega\left(d_{k, l}^{H}\right)$ : $\omega\left(C_{k, l}\right) \rightarrow \omega\left(C_{k, l+1}\right)$ sends $x \mapsto \omega(c) \wedge x$ where

$$
\omega(c):=\sum_{j=1}^{k}(\sum_{i=0}^{j}(-1)^{i} \underbrace{\uparrow_{\downarrow}^{e_{i}}}_{\uparrow} \underbrace{h_{j-i}}_{\downarrow}) \otimes w_{j} \in \operatorname{Dot}\left(\mathcal{E}^{(k)} \mathcal{F}^{(k)} \mathbf{1}_{n}\right) \otimes W_{k} .
$$

Similarly, the vertical differential is

$$
\begin{gathered}
\omega\left(d_{k, l}^{V}\right):=(-1)^{l} \\
\alpha_{k, l}^{\omega}\left(w_{i_{1}} \wedge w_{i_{2}} \wedge \cdots \wedge w_{i_{l}}\right) \\
=\mathbf{1} \otimes w_{i_{1}} \wedge \cdots \wedge w_{i_{l}}^{\alpha_{k, l}^{\omega}}: \omega\left(C_{k, l}\right) \rightarrow \omega\left(C_{k+1, l}\right) \text {, where } \\
-\sum_{j=1}^{l}(-1)^{k+1-i_{j}+l-j} \underbrace{\frac{e_{k+1-i_{j}}^{k+1}}{\downarrow}}_{\downarrow} \otimes\left(w_{i_{i}} \wedge \cdots \wedge \widehat{w}_{i_{j}} \wedge \cdots \wedge w_{i_{l}}\right) \wedge w_{k+1} .
\end{gathered}
$$

Since all the relations in $\dot{\mathcal{U}}$ are invariant under symmetries, $\omega\left(r \mathbf{1}_{-n}\right)$ is a bicomplex.

\subsection{The Image Under $\sigma$}

The bicomplex $\sigma\left(r \mathbf{1}_{-n}\right)$ has the same "chain groups" as $\omega\left(r \mathbf{1}_{-n}\right)$ (i.e., $\sigma\left(C_{k, l}\right)=\omega\left(C_{k, l}\right)$ ) with the differentials defined as follows: The horizontal differential $\sigma\left(d_{k, l}^{H}\right): \sigma\left(C_{k, l}\right) \rightarrow$ $\sigma\left(C_{k, l+1}\right)$ sends $x \mapsto \sigma(c) \wedge x$ where

$$
\sigma(c):=\sum_{j=1}^{k}\left(\sum_{i=0}^{j}(-1)^{i} \underset{\downarrow}{h_{j-i} k} \underset{\downarrow}{e_{i}^{k}}\right) \otimes w_{j} \in \operatorname{Dot}\left(\mathcal{E}^{(k)} \mathcal{F}^{(k)} \mathbf{1}_{n}\right) \otimes W_{k} .
$$

Similarly, the vertical differential is

$$
\sigma\left(d_{k, l}^{V}\right):=\left.(-1)^{l} \overbrace{k}^{\alpha_{k, l}}\right|_{k} ^{k+1}: \sigma\left(C_{k, l}\right) \rightarrow \sigma\left(C_{k+1, l}\right),
$$


where $\alpha_{k, l} \in \operatorname{Dot}\left(\mathcal{E}^{(k)} \mathbf{1}_{n}\right) \otimes \Lambda^{l} W_{k+1}$ is defined by (4.1).

\subsection{The Image Under $\psi$}

The 2-functor $\psi$ is contravariant on the 2-morphisms in $\dot{\mathcal{U}}$; hence, the bicomplex $\psi\left(r \mathbf{1}_{n}\right)$ looks as follows

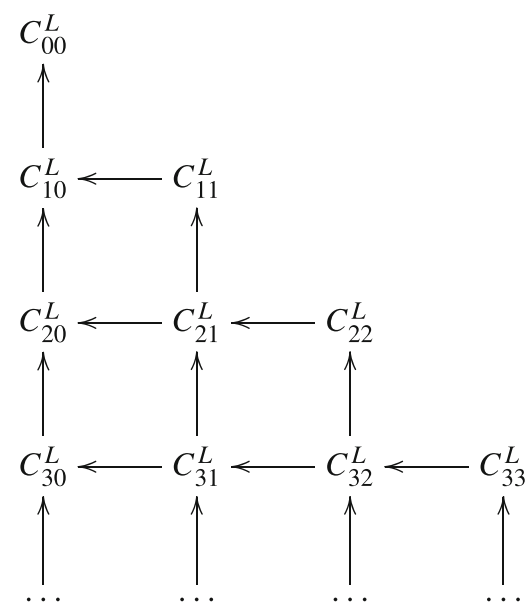

where the "chain groups" are

$$
C_{k, l}^{L}:=\psi\left(C_{k, l}\right)=\mathcal{F}^{(k)} \mathcal{E}^{(k)} \mathbf{1}_{n}\langle k n+k\rangle \otimes \Lambda^{l} \bar{W}_{k}
$$

with the total shifts $\left\langle\frac{n^{2}}{2}+n\right\rangle$ and $[-n / 2,-n / 2]$. The horizontal differential $\psi\left(d_{k, l}^{H}\right)$ : $C_{k, l+1}^{L} \rightarrow C_{k, l}^{L}$ sends

$$
\bar{w}_{i_{1}} \wedge \cdots \wedge \bar{w}_{i_{l+1}} \mapsto \sum_{j=1}^{l}(-1)^{j-1} c_{j} \otimes \bar{w}_{i_{1}} \wedge \widehat{\bar{w}}_{i_{j}} \wedge \bar{w}_{i_{l+1}},
$$

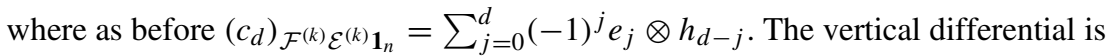

$$
\psi\left(d_{k, l}^{V}\right):=(-1)^{l}
$$

with $\alpha_{k, l}$ defined as before by (4.1).

It is easy to see that $\sigma \omega \psi\left(r \mathbf{1}_{n}\right)=r^{-1} \mathbf{1}_{n}$.

\section{Isomorphic Bicomplex}

This section provides a construction of the bicomplex $\tilde{r} \mathbf{1}_{n}$, which is isomorphic to the ribbon bicomplex and invariant under $\sigma \omega$. 


\subsection{The Isomorphism $H$}

Let $W=\operatorname{Span}_{\mathbb{Z}}\left\{w_{1}, w_{2}, \ldots\right\}$ and $W_{A}=A \otimes W$.

Then there exists an isomorphism $H: W_{A} \rightarrow W_{A}$ with

$$
H\left(w_{m}\right):=\sum_{j \geq m} h_{j-m} w_{j} .
$$

Its inverse is defined by replacing $h_{i}$ with its antipode $(-1)^{i} e_{i}$; hence, we have

$$
H^{-1}\left(w_{m}\right)=\sum_{j \geq m}(-1)^{j-m} e_{j-m} w_{j} .
$$

We can use this map to define a non-trivial transformation

$$
H_{k, l}: C_{k, l} \rightarrow C_{k, l}
$$

of the "chain groups" $C_{k, l}=\mathcal{F}^{(k)} \mathcal{E}^{(k)} \mathbf{1}_{n} \otimes \Lambda^{l} W_{k}$ of the ribbon bicomplex as follows.

For $l=1$, we set

$$
H_{k, 1}:=\left(\begin{array}{rrrrrr}
\mathbf{1} & 0 & 0 & 0 & \ldots & 0 \\
\left(h_{1}\right)_{2} & \mathbf{1} & 0 & 0 & \ldots & 0 \\
\left(h_{2}\right)_{2} & \left(h_{1}\right)_{2} & \mathbf{1} & 0 & \ldots & 0 \\
\left(h_{3}\right)_{2} & \left(h_{2}\right)_{2} & \left(h_{1}\right)_{2} & \mathbf{1} & \ldots & 0 \\
\ldots & \ldots & \ldots & \ldots & \ldots & 0 \\
\ldots & \ldots & \ldots &
\end{array}\right)=\left(\left(h_{i-j}\right)_{2}\right)_{1 \leq i, j \leq k},
$$

where the matrix is written in the basis $w_{1}, w_{2}, \ldots, w_{k}$ and all symmetric polynomials are sitting on the second strand.

This map obviously extends to $\Lambda^{l} W_{k}$ by setting

$$
\begin{aligned}
H_{k, l}\left(w_{i_{1}} \wedge w_{i_{2}} \wedge \cdots \wedge w_{i_{l}}\right): & =\left(\Lambda^{l} H_{k, 1}\right)\left(w_{i_{1}} \wedge w_{i_{2}} \wedge \cdots \wedge w_{i_{l}}\right) \\
& =H_{k, 1}\left(w_{i_{1}}\right) \wedge H_{k, 1}\left(w_{i_{2}}\right) \wedge \cdots \wedge H_{k, 1}\left(w_{i_{l}}\right) .
\end{aligned}
$$

Inserting (11.1), we get

$$
:=\sum_{1 \leq j_{1}<j_{2}<\cdots<j_{l} \leq k} \sum_{\sigma \in S_{l}}(-1)^{\sigma}\left(h_{j_{1}-i_{\sigma(1)}}\right)_{2}\left(w_{i_{1}} \wedge w_{i_{2}} \wedge \cdots \wedge w_{i_{l}}\right)
$$

Hence, for $1 \leq i_{1}<i_{2} \cdots<i_{l} \leq k$ and $1 \leq j_{1}<j_{2} \cdots<j_{l} \leq k$

$$
\left(H_{k, l}\right)_{j_{1}, j_{2}, \ldots, j_{l}}^{i_{1}, i_{2}, \ldots, i_{l}}=\sum_{\sigma \in S_{l}}(-1)^{\sigma}\left(h_{j_{1}-i_{\sigma(1)}}\right)_{2}\left(h_{j_{2}-i_{\sigma(2)}}\right)_{2} \ldots\left(h_{j_{l}-i_{\sigma(l)}}\right)_{2} .
$$

The inverse map is defined in a similar way by using

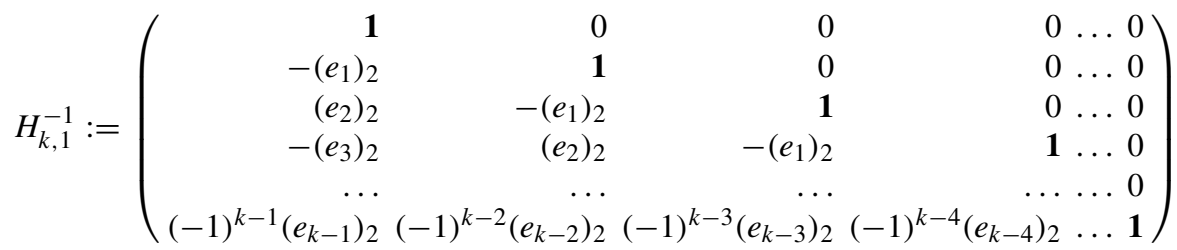

or

$$
\left(H_{k, 1}^{-1}\right)_{i, j}=\left((-1)^{i-j}\left(e_{i-j}\right)_{2}\right)_{1 \leq i, j \leq k} .
$$


For $1 \leq i_{1}<i_{2} \cdots<i_{l} \leq k$ and $1 \leq j_{1}<j_{2} \cdots<j_{l} \leq k$, we have

$$
\left(H_{k, l}^{-1}\right)_{j_{1}, j_{2}, \ldots, j_{l}}^{i_{1}, i_{2}, \ldots, i_{l}}=(-1)^{\sum_{s} i_{s}+j_{s}} \sum_{\sigma \in S_{l}}(-1)^{\sigma}\left(e_{j_{1}-i_{\sigma(1)}}\right)_{2}\left(e_{j_{2}-i_{\sigma(2)}}\right)_{2} \ldots\left(e_{j_{l}-i_{\sigma(l)}}\right)_{2} .
$$

\subsection{The Bicomplex $\tilde{r} 1_{n}$}

Let us denote by $\tilde{\boldsymbol{r}} \mathbf{1}_{n}$ the image of $r \mathbf{1}_{n}$ under applying the isomorphism $H_{k, l}$ to each "chain group" $C_{k, l}$. The horizontal and vertical differentials of $\tilde{r} \mathbf{1}_{n}$ are given by

$$
\tilde{d}_{k, l}^{H}:=H_{k, l+1}^{-1} d_{k, l}^{H} H_{k, l}, \quad \tilde{d}_{k, l}^{V}=H_{k+1, l}^{-1} d_{k, l}^{V} H_{k, l} .
$$

Let us compute them.

We start with $\tilde{d}_{k, 0}^{H}=H_{k, 1}^{-1} d_{k, 0}^{H}=H_{k, 1}^{-1} \sum_{i=1}^{k} c_{i} w_{i}$. Thus, the $i$ th entry of $\tilde{d}_{k, 0}^{H}$ is

$$
\begin{aligned}
\sum_{j=0}^{i-1}(-1)^{i-j}\left(e_{i-j}\right)_{2} c_{j} & =\left(c_{i}\right)_{1}-(-1)^{i}\left(e_{i}\right)_{2} \\
& =(-1)^{i}\left(\left(e_{i}\right)_{1}-\left(e_{i}\right)_{2}\right)=: y_{i} \in \operatorname{Dot}\left(\mathcal{F}^{(k)} \mathcal{E}^{(k)} \mathbf{1}_{n}\right) .
\end{aligned}
$$

In the general case,

$$
\begin{aligned}
\tilde{d}_{k, l}^{H}\left(w_{i_{1}} \wedge \cdots \wedge w_{i_{l}}\right) & =\left(\Lambda^{l+1} H_{k, 1}\right)^{-1}\left(c \wedge\left(\Lambda^{l} H_{k, 1}\right)\left(w_{i_{1}} \wedge \cdots \wedge w_{i_{l}}\right)\right) \\
& =\left(H_{k, 1}^{-1} c\right) \wedge w_{i_{1}} \wedge \cdots \wedge w_{i_{l}} \\
& =\sum_{i=1}^{k} y_{i} w_{i} \wedge w_{i_{1}} \wedge \cdots \wedge w_{i_{l}},
\end{aligned}
$$

where we used the centrality of $c$ and the previous computation. Observe that $\tilde{d}_{k, l}^{H}=$ $\sigma \omega\left(\tilde{d}_{k, l}^{H}\right)$.

To compute the vertical differential, we will need the following notation:

$$
t_{p}:=\left.\overbrace{p}^{k+1}\right|_{k} ^{k+1}
$$

Then it is easy to verify that $\left(e_{j}\right)_{2} t_{0}=t_{0}\left(e_{j}\right)_{2}+t_{1}\left(e_{j-1}\right)_{2}$ or more generally

$$
\left(e_{m_{1}} \ldots e_{m_{l}}\right)_{2} t_{0}=\sum_{a=0}^{l} t_{a} \sum_{\substack{T \subset\{1,2, \ldots, l\} \\|T|=a}}\left(e_{m_{1}-\varepsilon_{1}(T)} \ldots e_{m_{l}-\varepsilon_{l}(T)}\right)_{2},
$$

where $(x y)_{2}=(x)_{2}(y)_{2}$ and

$$
\varepsilon_{p}(T)= \begin{cases}1, & p \in T \\ 0, & p \notin T\end{cases}
$$

For instance

$$
\begin{aligned}
& \left(e_{m_{1}} e_{m_{2}} e_{m_{3}}\right)_{2} t_{0} \\
= & t_{0}\left(e_{m_{1}} e_{m_{2}} e_{m_{3}}\right)_{2}+t_{1}\left(\left(e_{m_{1}-1} e_{m_{2}} e_{m_{3}}\right)_{2}+\left(e_{m_{1}} e_{m_{2}-1} e_{m_{3}}\right)_{2}+\left(e_{m_{1}} e_{m_{2}} e_{m_{3}-1}\right)_{2}\right) \\
& +t_{2}\left(\left(e_{m_{1}-1} e_{m_{2}-1} e_{m_{3}}\right)_{2}+\left(e_{m_{1}-1} e_{m_{2}} e_{m_{3}-1}\right)_{2}+\left(e_{m_{1}} e_{m_{2}-1} e_{m_{3}-1}\right)_{2}\right) \\
& +t_{3}\left(e_{m_{1}-1} e_{m_{2}-1} e_{m_{3}-1}\right)_{2} .
\end{aligned}
$$


For simplicity, we put $t_{0}=t$. With this notation, let us first compute the matrix for the vertical differential in the case when $l=1$. Inserting (11.1), (11.2) into (11.3), we get

$$
\left(d_{k, 1}^{V}\right)_{i, j}= \begin{cases}-\sum_{p=0}^{i-j}(-1)^{p}\left(e_{p}\right)_{2} t\left(h_{i-j-p}\right)_{2}=-t \delta_{i-j, 0}+t_{1} \delta_{i-j, 1}, & i-j \geq 0 \\ 0 & \text { otherwise }\end{cases}
$$

where $\delta_{i, j}$ is the Kronecker delta-function. For example, for $k=3$, we have

$$
d_{k, 1}^{V}=\left(\begin{array}{rrrr}
\mathbf{1} & 0 & 0 & 0 \\
-\left(e_{1}\right)_{2} & \mathbf{1} & 0 & 0 \\
\left(e_{2}\right)_{2} & -\left(e_{1}\right)_{2} & \mathbf{1} & 0 \\
-\left(e_{3}\right)_{2} & \left(e_{2}\right)_{2} & -\left(e_{1}\right)_{2} & \mathbf{1}
\end{array}\right)(-t)\left(\begin{array}{rrr}
\mathbf{1} & 0 & 0 \\
0 & \mathbf{1} & 0 \\
0 & 0 & \mathbf{1} \\
\left(e_{3}\right)_{2} & -\left(e_{2}\right)_{2} & \left(e_{1}\right)_{2}
\end{array}\right)\left(\begin{array}{rrr}
\mathbf{1} & 0 & 0 \\
\left(h_{1}\right)_{2} & \mathbf{1} & 0 \\
\left(h_{2}\right)_{2} & \left(h_{1}\right)_{2} & \mathbf{1}
\end{array}\right)
$$

In general, for $1 \leq i_{1}<i_{2} \cdots<i_{l} \leq k$ and $1 \leq j_{1}<j_{2} \cdots<j_{l} \leq k$ using the computation for $l=1$ case, we get

$$
\begin{aligned}
\left(d_{k, l}^{V}\right)_{j_{1}, \ldots, j_{l}}^{i_{1}, \ldots, i_{l}} & =(-1)^{l} \sum_{1 \leq p_{1}<\cdots<p_{l} \leq k} \sum_{1 \leq s_{1}<\cdots<s_{l} \leq k+1}\left(H_{k+1, l}^{-1}\right)_{j_{1}, \ldots, j_{l}}^{s_{1}, \ldots, s_{l}} t\left(\alpha_{k, l}\right)_{s_{1}, \ldots, s_{l}}^{p_{1}, \ldots, p_{l}}\left(H_{k, l}\right)_{p_{1}, \ldots, p_{l}}^{i_{1}, \ldots, i_{l}} \\
& =\sum_{1 \leq p_{1}<\cdots<p_{l} \leq k+1}\left(H_{k+1, l}^{-1}\right)_{j_{1}, \ldots, j_{l}}^{p_{1}, \ldots, p_{l}} t \quad\left(H_{k+1, l}\right)_{p_{1}, \ldots, p_{l}}^{i_{1}, \ldots, i_{l}} .
\end{aligned}
$$

By (11.4), we obtain

$$
\begin{aligned}
\left(H_{k+1, l}^{-1}\right)_{j_{1}, \ldots, j_{l}}^{p_{1}, \ldots, p_{l}} t & =\sum_{a=0}^{l} t_{a}(-1)^{\sum_{s}\left(p_{s}-j_{s}\right)} \sum_{\sigma \in S_{l}}(-1)^{\sigma} \sum_{\substack{T \subset\{1,2, \ldots, l\} \\
|T|=a}} \prod_{\substack{l \\
\mid l=1}}^{l} e_{j_{s}-k_{\sigma(s)}-\varepsilon_{s}(T)} \\
& =\sum_{a=0}^{l}(-1)^{a} t_{a} \sum_{\substack{T \subset\{1,2, \ldots, l\} \\
|T|=a}}\left(H_{k+1, l}^{-1}\right)_{\substack{j_{1}^{T}, \ldots, j_{l}^{T} \\
p_{1}, \ldots, p_{l}}}
\end{aligned}
$$

where $j_{s}^{T}:=j_{s}-\varepsilon_{s}(T)$. Hence

$$
\begin{aligned}
& \left(d_{k, l}^{V}\right)^{i_{1}, \ldots, i_{l}} j_{1}, \ldots, j_{l} \\
= & (-1)^{l} \sum_{a=0}^{l}(-1)^{a} t_{a} \sum_{\substack{T \subset\{1,2, \ldots, l\} \\
|T|=a}} \prod_{s=1}^{l} \delta_{j_{s}^{T}, i_{s}} \\
= & \begin{cases}(-1)^{l+a} t_{a} & \text { if }\left(j_{1}, \ldots, j_{l}\right) \text { is obtained from }\left(i_{1}, \ldots, i_{l}\right) \text { by shifting } a \text { entries by }-1 \\
0 & \text { otherwise. }\end{cases}
\end{aligned}
$$

Observe that $\sigma \omega\left(t_{a}\right)=t_{a}$ and hence $d_{k, l}^{V}=\sigma \omega\left(d_{k, l}^{V}\right)$. 


\section{Proof of the Invertibility of the Ribbon Complex}

\subsection{The 2-functor $\Gamma_{N}$}

Let us recall the definition of the 2-functor $\Gamma_{N}$ from [12, Section 7]. On objects the 2-functor $\Gamma_{N}$ sends $n$ to the ring $H_{k ; N}$ whenever $n$ and $k$ are compatible

$$
\begin{aligned}
\Gamma_{N}: \mathcal{U} & \rightarrow \operatorname{Flag}_{N} \\
n & \mapsto \begin{cases}H_{k ; N} & \text { with } n=2 k-N \text { and } 0 \leq k \leq N, \\
0 & \text { otherwise. }\end{cases}
\end{aligned}
$$

1-Morphisms of $\mathcal{U}$ get mapped by $\Gamma_{N}$ to graded bimodules

$$
\begin{aligned}
\Gamma_{N}: \mathcal{U} & \rightarrow \text { Flag }_{N} \\
\mathbf{1}_{n}\langle s\rangle & \mapsto \begin{cases}H_{k ; N}\langle s\rangle & \text { with } n=2 k-N \text { and } 0 \leq k \leq N, \\
0 & \text { otherwise. }\end{cases} \\
\mathcal{E} \mathbf{1}_{n}\langle s\rangle & \mapsto \begin{cases}H_{k+1, k ; N}\langle s+1-N+k\rangle & \text { with } n=2 k-N \text { and } 0 \leq k<N, \\
0 & \text { otherwise. }\end{cases} \\
\mathcal{F} \mathbf{1}_{n}\langle s\rangle & \mapsto \begin{cases}H_{k-1, k ; N}\langle s+1-k\rangle & \text { with } n=2 k-N \text { and } 0<k \leq N, \\
0 & \text { otherwise, }\end{cases}
\end{aligned}
$$

where the cohomology of the Grassmannian is given by

$$
H_{k ; N}:=\mathbb{Z}\left[x_{1, n}, x_{2, n}, \ldots, x_{k, n} ; y_{1, n}, \ldots, y_{N-k, n}\right] / I_{k ; N}
$$

with $I_{k ; N}$ the homogeneous ideal generated by elements equating powers of $t$ in the equation

$$
\left(1+x_{1, n} t+\cdots+x_{k, n} t^{k}\right)\left(1+y_{1, n} t+\cdots+y_{N-k, n} t^{N-k}\right)=1 .
$$

The cohomology of the $a$ th iterated 1 -step flag variety $H_{\underline{k} ; N}$ with $\underline{k}=(k, k+1, k+$ $2, \ldots, k+a)$ is given by

$$
H_{\underline{k} ; N}:=\mathbb{Z}\left[x_{1, n}, x_{2, n}, \ldots, x_{k, n} ; \xi_{1}, \ldots, \xi_{a} ; y_{1, n+2 a}, \ldots, y_{N-k-a, n+2 a}\right] / I_{\underline{k} ; N}
$$

with $I_{\underline{k} ; N}$ the homogeneous ideal generated by elements equating powers of $t$ in the equation

$$
\begin{aligned}
& \left(1+x_{1, n} t+\cdots+x_{k, n} t^{k}\right)\left(1+\xi_{1} t\right)\left(1+\xi_{2} t\right) \ldots\left(1+\xi_{a} t\right) \\
& \left(1+y_{1, n+2 a} t+\cdots+y_{N-k-a, n+2 a} t^{N-k-a}\right)=1 .
\end{aligned}
$$

We will also use the cohomology of $a$-step flag variety corresponding to the sequence $\underline{k}=(k, k+a)$ given by

$$
H_{k, k+a ; N}:=\mathbb{Z}\left[x_{1, n}, x_{2, n}, \ldots, x_{k, n} ; \varepsilon_{1}, \ldots, \varepsilon_{a} ; y_{1, n+2 a}, \ldots, y_{N-k-a, n+2 a}\right] / I_{k, k+a ; N}
$$

with $I_{k, k+a ; N}$ the homogeneous ideal generated by elements equating powers of $t$ in the equation

$$
\begin{gathered}
\left(1+x_{1, n} t+\cdots+x_{k, n} t^{k}\right)\left(1+\varepsilon_{1} t+\cdots+\varepsilon_{a} t^{a}\right) \\
\left(1+y_{1, n+2 a} t+\cdots+y_{N-k-a, n+2 a} t^{N-k-a}\right)=1 .
\end{gathered}
$$


It will be convenient in what follows to introduce a simplified notation in Flag $_{N}$. Corresponding to a fixed value of $N$, we set $n=2 k-N$ and write

$$
\begin{aligned}
\mathbb{1}_{n}^{N} & :=\Gamma_{N}\left(\mathbf{1}_{n}\right) \\
\mathrm{E} \mathbb{1}_{n}^{N} & =\mathbb{1}_{n+2}^{N} \mathrm{E}=\mathbb{1}_{n+2}^{N} \mathrm{E} \mathbb{1}_{n}^{N}:=\Gamma_{N}\left(\mathcal{E} \mathbf{1}_{n}\right) \\
\mathrm{F} \mathbb{1}_{n}^{N} & =\mathbb{1}_{n-2}^{N} \mathrm{~F}=\mathbb{1}_{n-2}^{N} \mathrm{~F} \mathbb{1}_{n}^{N}:=\Gamma_{N}\left(\mathcal{F} \mathbf{1}_{n}\right)
\end{aligned}
$$

as a shorthand for the various bimodules. Juxtaposition of these symbols represents the tensor product of the corresponding bimodules. For example

$$
\text { FEE } \mathbb{1}_{n}^{N}=H_{k+1, k+2 ; N} \otimes_{H_{k+2 ; N}} H_{k+2, k+1 ; N} \otimes_{H_{k+1 ; N}} H_{k+1, k ; N} .
$$

Associated with a signed sequence $\underline{\epsilon}$ is the $\left(H_{k+\mid \underline{\epsilon}}, H_{k}\right)$-bimodule

$$
\mathrm{E}_{\underline{\epsilon}} \mathbb{1}_{n}^{N}:=\mathrm{E}_{\epsilon_{1}} \mathrm{E}_{\epsilon_{2}} \ldots \mathrm{E}_{\epsilon_{m}} \mathbb{1}_{n}^{N}
$$

where $\mathrm{E}_{+}:=\mathrm{E}$ and $\mathrm{E}_{-}:=\mathrm{F}$. The 2-functor $\Gamma_{N}$ maps a composite $\mathcal{E}_{\underline{\epsilon}} \mathbf{1}_{n}$ of 1-morphisms in $\dot{\mathcal{U}}$ to the tensor product $\mathrm{E}_{\epsilon} 1_{n}^{N}$ in $\mathbf{F l a g}_{N}$. Note that because tensor product of bimodules is only associative up to coherent isomorphism, our notation is ambiguous unless we choose a parenthesization of the bimodules in question. We employ the convention that all parenthesis are on the far left. Hence, $\Gamma_{N}$ preserves composition of 1-morphisms only up to coherent 2-isomorphism.

It is sometimes convenient to use the following isomorphisms from [3].

$$
\begin{array}{ll}
\Gamma_{N}\left(\mathcal{E}^{a} \mathbf{1}_{n}\right) \cong H_{k+a, k+a-1, \ldots, k ; N}\left\langle r_{a}\right\rangle, & r_{a}=\sum_{i=1}^{a} i-N+k \\
\Gamma_{N}\left(\mathcal{F}^{a} \mathbf{1}_{n}\right) \cong H_{k, k+1, \ldots, k+a ; N}\left\langle r_{a}^{\prime}\right\rangle, & r_{a}^{\prime}=\sum_{i=1}^{a} i-k .
\end{array}
$$

We also define bimodules

$$
\begin{aligned}
\mathrm{E}^{(a)} \mathbb{1}_{n}^{N}:=H_{k+a, k ; N}\left\langle r_{a}-\frac{a(a-1)}{2}\right\rangle, \\
\mathrm{F}^{(b)} \mathbb{1}_{n}^{N}:=H_{k+a, k ; N}\left\langle r_{a}+\frac{a(a-1)}{2}\right\rangle .
\end{aligned}
$$

Let us denote by $r \mathbb{1}_{n}^{N}$ the image under $\Gamma_{N}$ of the ribbon complex.

\subsection{Proof of Theorem 1.5}

We first prove that tensoring on the left (or on the right) with $r \mathbb{1}_{n}^{N}$ acts as a left (resp. right) multiplication with the identity on any left (resp. right) $H_{k ; N}$-module up to degree shift.

We will consider the left action only, the right action can be proved similarly. Note that it is enough to compute the left action of $r \mathbb{1}_{n}^{N}$ on $H_{k ; N}$. Let us first ignore the homological shift for simplicity.

Set $n=N$. Then $r \mathbb{1}_{N}^{N}=\mathbb{1}_{N}^{N}\left\langle-N^{2} / 2-N\right\rangle$ in $\operatorname{Kom}\left(\mathbf{F l a g}_{N}\right)$, simply because $\mathrm{E} \mathbb{1}_{N}^{N}=0$, and the result holds.

Assume $n=N-2 k$, then

$$
\mathrm{rF}^{k} \mathbb{1}_{N}^{N} \simeq \mathrm{F}^{k} \mathrm{r} \mathbb{1}_{N}^{N}=\mathrm{F}^{k} \mathbb{1}_{N}^{N}\left\langle-N^{2} / 2-N\right\rangle,
$$


where the first homotopy equivalence holds due to centrality of $r \mathbb{1}_{n}^{N}$. Now observe that:

$$
\mathrm{F}^{k} \mathbb{1}_{N}^{N} \simeq \bigoplus_{[k] !} \mathrm{F}^{(k)} \mathbb{1}_{N}^{N}
$$

in $\mathbf{F l a g}_{N}$. Hence, $r \mathbb{1}_{n}^{N}$ acts as a left multiplication with $\mathbb{1}_{n}^{N}\left\langle-N^{2} / 2-N\right\rangle$ on $\mathrm{F}^{(k)} \mathbb{1}_{N}^{N}$ in $\operatorname{Com}\left(\operatorname{Flag}_{N}\right)$. But $\mathrm{F}^{(k)} \mathbb{1}_{N}^{N}$ is isomorphic to $H_{k ; N}=\mathbb{1}_{n}^{N}$ as a left module over itself. Hence we have the first statement.

Similarly, tensoring with $r^{-1} \mathbb{1}_{n}^{N}$ on the left and on the right is homotopic to the identity functor shifted by $\left\langle N^{2} / 2+N\right\rangle$, which is inverse to $r \mathbb{1}_{n}^{N}$. Since homological shifts for $r \mathbf{1}_{n}$ and $r^{-1} \mathbf{1}_{n}$ are inverse to each other, we have the result.

\subsection{The Inverse Limit of Schur Quotients}

The Schur quotient $\dot{\mathcal{U}}_{N}$ of $\dot{\mathcal{U}}$ is defined by setting $\mathbf{1}_{N+2}=0$ (see [14] for a more general definition). Applying sl(2) relations, an easy induction argument shows that $\mathbf{1}_{n}=0$ in $\dot{\mathcal{U}}_{N}$ for all $n<-N$ and $n>N$. Moreover, this quotient is not empty, since the functor $\Gamma_{N}: \dot{\mathcal{U}} \rightarrow$ Flag $_{N}$ factorizes through $\dot{\mathcal{U}}_{N}$ by its very definition.

For any $N^{\prime}>N$, there is a natural projection

$$
\tilde{\Psi}_{N^{\prime}, N}: \dot{\mathcal{U}}_{N^{\prime}} \rightarrow \dot{\mathcal{U}}_{N}
$$

defined by setting $\mathbf{1}_{N+2}=0$. Taking all together, these maps define an inverse system of 2-categories whose inverse limit is $\dot{\mathcal{U}}$ (compare [3]).

Now let us consider $\operatorname{Com}^{b}\left(\dot{\mathcal{U}}_{N}\right)$. The induced functor $\operatorname{Com}^{b}(\dot{\mathcal{U}}) \rightarrow \operatorname{Com}^{b}\left(\operatorname{Flag}_{N}\right)$ factorizes again through $\operatorname{Com}^{b}\left(\dot{\mathcal{U}}_{N}\right)$. Using the natural projections

$$
\Psi_{N^{\prime}, N}: \operatorname{Com}^{b}\left(\dot{\mathcal{U}}_{N^{\prime}}\right) \rightarrow \operatorname{Com}^{b}\left(\dot{\mathcal{U}}_{N}\right)
$$

for all $N^{\prime}>N$, we can construct

$$
{ }^{l} \operatorname{Com}(\dot{\mathcal{U}})=\lim _{\leftarrow} \operatorname{Com}^{b}\left(\dot{\mathcal{U}}_{N}\right)
$$

Observe that $r^{ \pm 1} r^{\mp 1} \mathbf{1}_{n}$ belongs to ${ }^{l} \operatorname{Com}(\dot{\mathcal{U}})$, since its projections to $\operatorname{Com}^{b}\left(\dot{\mathcal{U}}_{N}\right)$ are welldefined for all $N$ and compatible with each other.

\subsection{Proof of Theorem 1.1 (Invertibility)}

By the universal property of the inverse 2-limit, we get a 2-functor $\widehat{\Gamma}:{ }^{l} \operatorname{Com}(\dot{\mathcal{U}}) \rightarrow \lim \operatorname{Com}^{b}$ (Flag), unique up to 2-isomorphism in iBicat, making the diagram

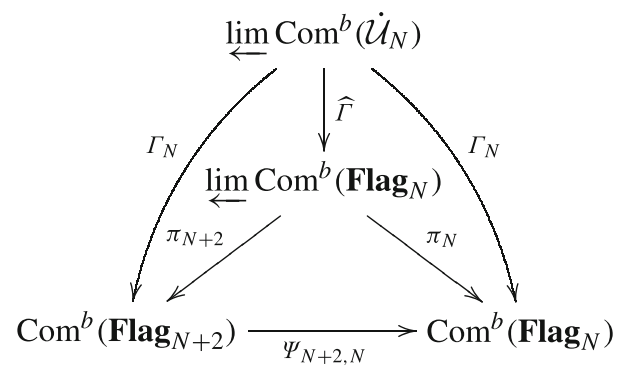

commute up to 2-isomorphism in iBicat. Note that iBicat is a bicategory with objects bicategories, morphisms (pseudo) 2-functors and 2-morphisms given by icons. 
Now the arguments in [3] (e.g., proof of Theorem 3.2) imply that this 2-functor $\widehat{\Gamma}:{ }^{l} \operatorname{Com}(\dot{\mathcal{U}}) \rightarrow \lim \operatorname{Com}^{b}($ Flag) is an equivalence of 2 -categories in iBicat. Since $r r^{-1} \mathbf{1}_{n}=\widehat{\Gamma}^{-1}\left(\lim _{\leftarrow}^{\leftarrow} \mathbb{1}_{n}^{N}\right)$ by Theorem 1.5 , we get the desired result.

Acknowledgments The first author would like to thank Aaron Lauda for helpful discussions and Krzysztof Putyra for sharing his LaTeX package for drawing diagrams.

Funding Open access funding provided by University of Zurich.

\section{Appendix}

Let us collect the identities we need in the proofs.

\section{Sliding Rules}

Generalizing the NilHecke algebra relations to the thick lines, we get
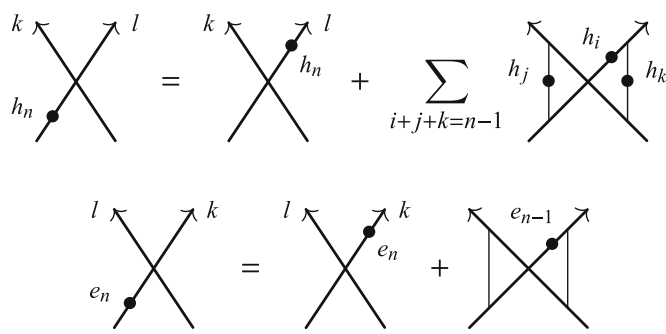

In particular, if $l=1$, we have

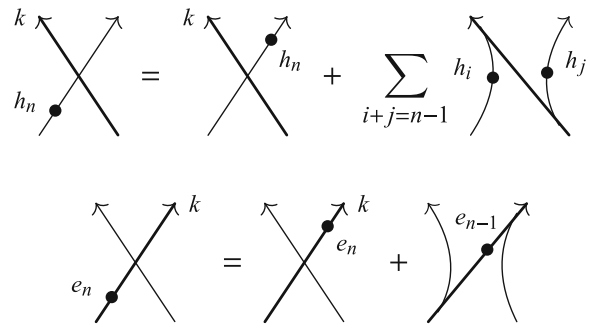

In what follows an $x$ labeled bullet on a thick line will mean $h_{x}$ inserted.

\section{Reidemeister Moves}

From [11, Corollary 5.8] (for $b=k, a=1$ ) and the bubble slide rule (4.11), we get

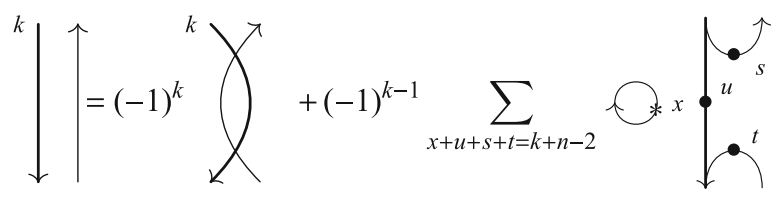


Analogously, [11, Theorem 5.6 and (4.12)] imply

$$
\uparrow=(-1)^{k} \bigcup^{k}+(-1)^{k-1} \sum_{x+u+s+t=k+n-2} O_{x} \overbrace{}^{u}
$$

Similar, to the proof of [12, Proposition 5.8], we can show that for all colors $n \in \mathbb{Z}$ of the right most region, the following equation holds:

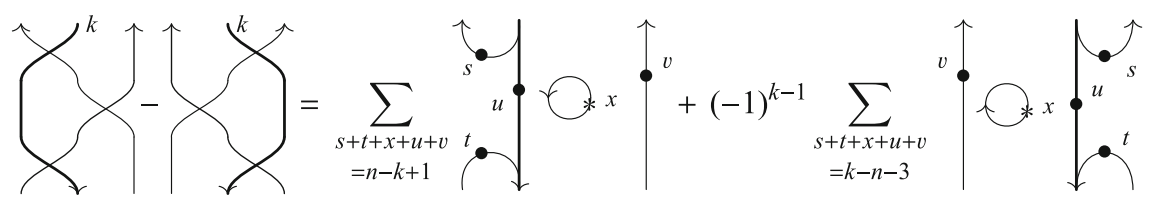

(A.6)

\section{Further Identities}

\section{Lemma A.1}

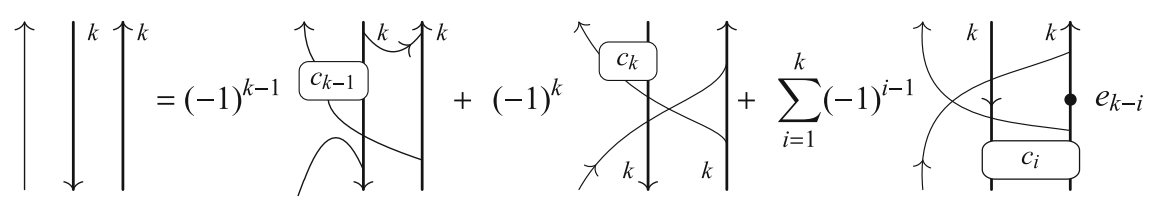

Proof We first simplify the last summand of this identity by using the fact that $c_{i}$ is central and

$$
\sum_{i=1}^{k}(-1)^{k-i} \overbrace{\substack{\downarrow \\ c_{i}}}^{\left.\right|_{k-1} ^{\dagger}} e_{k-i}=\left.\overbrace{k}^{\uparrow} \uparrow\right|_{k-1}
$$

Then we apply (A.6) to the second summand on the right hand side. Furthermore, we use

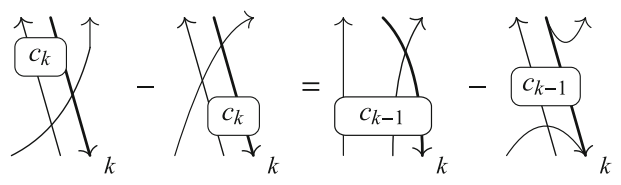

to simplify the resulting terms. The last equality follows from (A.3) to (A.4). It is easy to check that all terms with bubbles sum to zero. 
Lemma A.2 For $1 \leq i, j \leq k$, we have
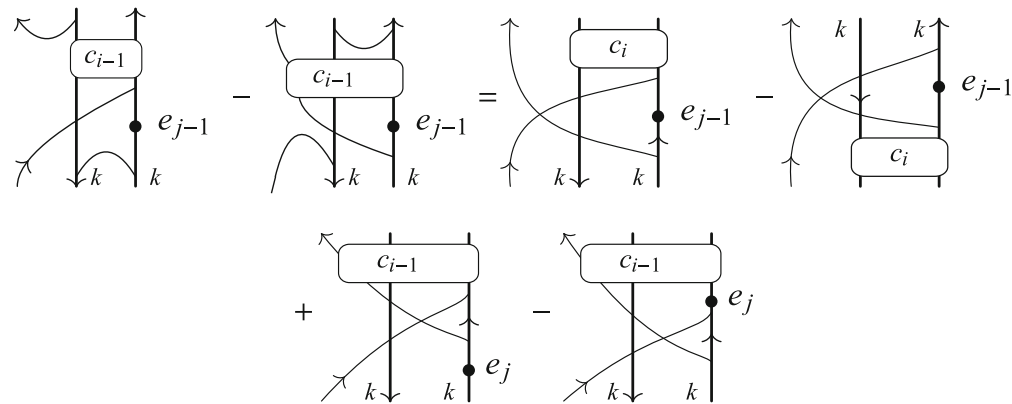

Proof We first apply (A.6) to the first two summands on the right hand side, then all diagrams without smoothings will look as shown below.

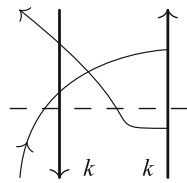

The strategy of the proof will be to move all dots into the position shown by the dashed line. Doing so for the last two summands, we get

$$
c_{i-1}\left(e_{1}\right)_{3}\left(e_{j-1}\right)_{4}-c_{i-1}\left(e_{1}\right)_{1}\left(e_{j-1}\right)_{4} \in \operatorname{Dot}\left(\mathcal{E} \mathcal{F}^{(k)} \mathcal{E} \mathcal{E}^{(k-1)}\right)
$$

minus the second term on the left hand side of the identity (obtained after sliding the dot though the down pointed $k$-line), and in addition various terms with bubbles. Let us first compare the terms without smoothings. The second term on the right hand side will contribute:

$$
-\left(c_{i}\right)_{234}\left(e_{j-1}\right)_{4} \in \operatorname{Dot}\left(\mathcal{E} \mathcal{F}^{(k)} \mathcal{E} \mathcal{E}^{(k-1)}\right) .
$$

Finally, in the first term on the right hand side, we replace $\left(c_{i}\right)_{23}$ with $c_{i}-c_{i-1}\left(e_{1}\right)_{1}$ and move the dot down. The contribution of this term to the part without smoothings will be

$$
c_{i}\left(e_{j-1}\right)_{4}-c_{i-1}\left(e_{1}\right)_{3}\left(e_{j-1}\right)_{4} \in \operatorname{Dot}\left(\mathcal{E} \mathcal{F}^{(k)} \mathcal{E} \mathcal{E}^{(k-1)}\right)
$$

and in addition from moving the dot, we get the first term on the left hand side. Collecting all non-smoothed terms together we get zero. It remains to show that all bubble terms vanish. This easy check is left to the reader.

Open Access This article is licensed under a Creative Commons Attribution 4.0 International License, which permits use, sharing, adaptation, distribution and reproduction in any medium or format, as long as you give appropriate credit to the original author(s) and the source, provide a link to the Creative Commons licence, and indicate if changes were made. The images or other third party material in this article are included in the article's Creative Commons licence, unless indicated otherwise in a credit line to the material. If material is not included in the article's Creative Commons licence and your intended use is not permitted by statutory regulation or exceeds the permitted use, you will need to obtain permission directly from the copyright holder. To view a copy of this licence, visit http://creativecommonshorg/licenses/by/4.0/.

\section{References}

1. Beilinson, A., Lusztig, G., MacPherson, R.: A geometric setting for the quantum deformation of $\mathrm{GL}_{n}$. Duke. Math. J. 61(2), 655-677 (1990) 
2. Beliakova, A., Khovanov, M., Lauda, A.D.: A categorification of the Casimir of quantum sl(2). Adv. Math. 230(3), 1442-1501 (2012)

3. Beliakova, A., Lauda, A.: Categorified quantum sl(2) is an inverse limit of Flag 2-categories. Transform. Groups 19, 1-26 (2014)

4. Cautis, S.: Clasp technology to knot homology via the affine Grassmannian. Math. Ann. 363, 1053-1115 (2015)

5. Cautis, S., Kamnitzer, J.: Braiding via geometric categorical Lie algebra actions. Compositio Math. 148, 464-506 (2012)

6. Chuang, J., Rouquier, R.: Derived equivalences for symmetric groups and $s l_{2}$-categorification. Ann. of Math. 167, 245-298 (2008)

7. Habiro, K.: A unified Witten-Reshetikhin-Turaev invariant for integral homology spheres. Invent. Math. 171(1), 1-81 (2008)

8. Khovanov, M., Lauda, A.: A diagrammatic approach to categorification of quantum groups I. Represent. Theory 13, 309-347 (2009)

9. Khovanov, M., Lauda, A.: A diagrammatic approach to categorification of quantum groups II. Trans. Am. Math. Soc. 363, 2685-2700 (2011)

10. Khovanov, M., Lauda, A.: A diagrammatic approach to categorification of quantum groups III. Quantum Topology 1, 1-92 (2010)

11. Khovanov, M., Lauda, A., Mackaay, M., Stošić, M.: Extended graphical calculus for categorified quantum sl(2). Memoirs of the AMS vol. 219 (2012)

12. Lauda, A.D.: A categorification of quantum sl(2). Adv. Math. 225, 3327-3424 (2008)

13. Lawrence, R.J.: A Universal Link Invariant. In: The interface of mathematics and particle physics (Oxford, 1988), Vol. 24 of Inst. Math. Appl. Conf. Ser. New Ser., pp 151-156. Oxford Univ. Press, New York (1990)

14. Mackaay, M., Stosic, M., Vaz, P.: A diagrammatic categorification of the $q$-Schur algebra. Quantum Topol. 4(1), 1-75 (2013)

15. Mazorchuk, V., Stroppel, C.: Projective-injective modules, Serre functors and symmetric algebras. J. Reine Angew. Math. 616, 131-165 (2008)

16. Rouquier, R.: 2-Kac-Moody algebras. arXiv:0812.5023

Publisher's Note Springer Nature remains neutral with regard to jurisdictional claims in published maps and institutional affiliations. 